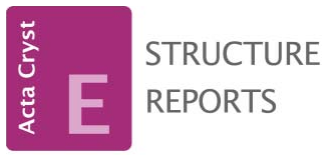

OPEN $\odot$ ACCESS

ISSN 1600-5368

\section{Crystal structure of (pyridin-2-ylmethyl- idene)(triphenylmethyl)amine}

\section{Chatphorn Theppitak, ${ }^{a}$ Manlika Meesangkaew, ${ }^{a}$ Songwuit Chanthee, ${ }^{a}$ Nimit Sriprang ${ }^{a}$ and Kittipong Chainok ${ }^{b}$ *}

a Department of Chemistry, Faculty of Science, Naresuan University, Mueang, Phitsanulok 65000, Thailand, and ${ }^{\mathbf{b}}$ Department of Physics, Faculty of Science and Technology, Thammasat University, Khlong Luang, Pathum Thani 12120, Thailand.

*Correspondence e-mail: kc@tu.ac.th

Received 28 August 2014; accepted 29 August 2014
The title Schiff base compound, $\mathrm{C}_{25} \mathrm{H}_{20} \mathrm{~N}_{2}$, crystallizes with two independent molecules $(A$ and $B)$ in the asymmetric unit. In both molecules, the imine group is approximately coplanar with the pyridine ring, with $\mathrm{N}-\mathrm{C}-\mathrm{C}-\mathrm{N}$ torsion angles of 170.1 (3) and -172.0 (3) $\AA$. In the crystal, $A$ and $B$ dimers are linked by pairs of $\mathrm{C}-\mathrm{H} \cdots \pi$ interactions and further $\mathrm{C}-$ $\mathrm{H} \cdots \pi$ bonds link the dimers into a three-dimensional network.

Keywords: crystal structure; amine; pyridin-2-ylmethylidene; trityl; Schiff base ligands; magnetism.

CCDC reference: 1021733

\section{Related literature}

For the use of the pyridin-2-ylmethanimine Schiff base ligands for the development of a new generation of memory devices, multifunctional materials, and other magnetic applications, see: Capes et al. (2000); Guionneau et al. (2001); Létard et al. (1997, 1998); Liu et al. (2010); Murray (2008); Goodwin (2004); Gupta \& Sutar (2008). For van der Waals radii, see: Bondi (1964)

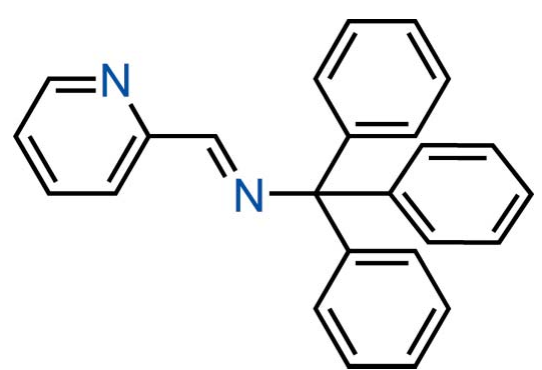

\section{Experimental}

\subsection{Crystal data}

\section{$\mathrm{C}_{25} \mathrm{H}_{20} \mathrm{~N}_{2}$}

$M_{r}=348.43$

Orthorhombic, $P 2_{1} 2{ }_{1} 2_{1}$

$a=7.0719(5) \AA$

$b=16.3972(8) \AA$

$c=31.880(2) \AA$

$$
\begin{aligned}
& V=3696.7(4) \AA^{3} \\
& Z=8 \\
& \text { Cu K } \alpha \text { radiation } \\
& \mu=0.56 \mathrm{~mm}^{-1} \\
& T=173 \mathrm{~K} \\
& 0.30 \times 0.26 \times 0.22 \mathrm{~mm}
\end{aligned}
$$

\subsection{Data collection}

Agilent Xcalibur (Sapphire3, Gemini ultra) diffractometer

Absorption correction: multi-scan (CrysAlis PRO and CrysAlis RED; Agilent, 2012) $T_{\min }=0.849, T_{\max }=0.886$

21735 measured reflections 6965 independent reflections 5059 reflections with $I>2 \sigma(I)$ $R_{\text {int }}=0.058$

\subsection{Refinement}

$R\left[F^{2}>2 \sigma\left(F^{2}\right)\right]=0.057$

$w R\left(F^{2}\right)=0.153$

$S=1.00$

6965 reflections

487 parameters

$\mathrm{H}$-atom parameters constrained

$\Delta \rho_{\max }=0.36 \mathrm{e} \AA^{-3}$

$\Delta \rho_{\min }=-0.18 \mathrm{e} \AA^{-3}$

Table 1

Hydrogen-bond geometry $\left(\AA{ }^{\circ}\right)$.

$C g 1$ is the centroid of the N1, C1-C5 ring, $C g 2$ of ring C8-C13, $C g 3$ of ring $\mathrm{C} 14-\mathrm{C} 19, C g 4$ of ring $\mathrm{C} 20-\mathrm{C} 25, C g 5$ of ring N1 ${ }^{\prime}, \mathrm{C1}^{\prime}-\mathrm{C}^{\prime}, C g 6$ of ring $\mathrm{C}^{\prime}-\mathrm{C}^{\prime} 3^{\prime}$, $\mathrm{Cg} 7$ of ring $\mathrm{C} 14^{\prime}-\mathrm{C} 19^{\prime}$ and $\mathrm{Cg} 8$ of ring $\mathrm{C} 20^{\prime}-\mathrm{C} 25^{\prime}$.

\begin{tabular}{llllll}
\hline $\mathrm{D}-\mathrm{H} \cdots \mathrm{A}$ & $\mathrm{D}-\mathrm{H}$ & $\mathrm{H} \cdots \mathrm{A}$ & $\mathrm{H} \cdots P l a n e$ & $\mathrm{D} \cdots \mathrm{A}$ & $\mathrm{D}-\mathrm{H} \cdots \mathrm{A}$ \\
\hline $\mathrm{C} 1-\mathrm{H} 1 \cdots C g 8$ & 0.95 & 2.819 & $2.786(3)$ & 3.573 & 137 \\
$\mathrm{C} 1^{\prime}-\mathrm{H} 1^{\prime} \cdots C g 4$ & 0.95 & 2.789 & $2.749(3)$ & 3.545 & 137 \\
$\mathrm{C} 2-\mathrm{H} 2 \cdots C g 5^{\mathrm{i}}$ & 0.95 & 2.769 & $2.751(3)$ & 3.591 & 145 \\
$\mathrm{C} 2^{\prime}-\mathrm{H} 2^{2} \cdots C g 1^{\text {ii }}$ & 0.95 & 2.789 & $2.717(3)$ & 3.555 & 138 \\
$\mathrm{C} 4-\mathrm{H} 4 \cdots C g 2^{\text {iii }}$ & 0.95 & 3.150 & $2.982(3)$ & 3.692 & 118 \\
$\mathrm{C} 4^{\prime}-\mathrm{H} 4^{\prime} \cdots C g 6^{\text {iv }}$ & 0.95 & 3.169 & $2.942(3)$ & 3.687 & 116 \\
$\mathrm{C} 9-\mathrm{H} 9 \cdots C g 4$ & 0.95 & 3.104 & $2.533(4)$ & 3.670 & 120 \\
$\mathrm{C} 9^{\prime}-\mathrm{H} 9^{\prime} \cdots C g 8$ & 0.95 & 3.061 & $2.526(4)$ & 3.642 & 121 \\
$\mathrm{C} 10-\mathrm{H} 10 \cdots C g 3^{\text {iv }}$ & 0.95 & 3.077 & $3.038(4)$ & 3.823 & 136 \\
$\mathrm{C} 10^{\prime}-\mathrm{H} 10^{\prime} \cdots C g 7^{\text {iii }}$ & 0.95 & 3.133 & $3.067(4)$ & 3.871 & 136 \\
$\mathrm{C} 15-\mathrm{H} 15 \cdots C g 2$ & 0.95 & 3.030 & $2.386(4)$ & 3.717 & 130 \\
$\mathrm{C} 15^{\prime}-15^{\prime} \cdots C g 6$ & 0.95 & 3.027 & $2.387(4)$ & 3.715 & 131 \\
$\mathrm{C} 21-\mathrm{H} 21 \cdots C g 3$ & 0.95 & 2.897 & $2.424(4)$ & 3.614 & 133 \\
$\mathrm{C} 21^{\prime}-\mathrm{H} 21^{\prime} \cdots C g 7$ & 0.95 & 2.886 & $2.435(4)$ & 3.604 & 133 \\
$\mathrm{C} 25-\mathrm{H} 25 \cdots C g 1^{\text {iv }}$ & 0.95 & 2.859 & $2.824(3)$ & 3.493 & 125 \\
$\mathrm{C} 25^{\prime}-\mathrm{H} 25^{\prime} \cdots C g 5^{\text {iii }}$ & 0.95 & 2.865 & $2.824(3)$ & 3.496 & 125 \\
\hline
\end{tabular}

Symmetry codes: (i) $1-x, \frac{1}{2}+y, \frac{3}{2}-z$; (ii) $-x, y-\frac{1}{2}, \frac{3}{2}-z$; (iii) $1+x, y, z$; (iv) $x-1, y, z$. 
Data collection: CrysAlis PRO (Agilent, 2012); cell refinement: CrysAlis PRO; data reduction: CrysAlis PRO; program(s) used to solve structure: SHELXS97 (Sheldrick, 2008); program(s) used to refine structure: SHELXL97 (Sheldrick, 2008); molecular graphics: ORTEP-3 for Windows (Farrugia, 2012) and DIAMOND (Brandenburg, 2006); software used to prepare material for publication: publCIF (Westrip, 2010).

\section{Acknowledgements}

We thank Professor Ian D. Williams and Dr Herman, H.-Y. Sung, Department of Chemistry, The Hong Kong University of Science and Technology, for help with the X-ray data collection. This research was supported by research career development grant (No. RSA5780056) from the Thailand Research Fund.

Supporting information for this paper is available from the IUCr electronic archives (Reference: TK5243).

\section{References}

Agilent (2012). CrysAlis PRO and CrysAlis RED. Agilent Technologies, Yarnton, England.

Bondi, A. (1964). J. Phys. Chem. 68, 441-451.

Brandenburg, K. (2006). DIAMOND. Crystal Impact GbR, Bonn, Germany.

Capes, L., Létard, J.-F. \& Kahn, O. (2000). Chem. Eur. J. 6, 2246-2255.

Farrugia, L. J. (2012). J. Appl. Cryst. 45, 849-854.

Goodwin, H. A. (2004). Top. Curr. Chem. 233, 59-90.

Guionneau, P., Brigouleix, C., Barrans, Y., Goeta, A. E., Létard, J.-F., Howard, J. A. K., Gaultier, J. \& Chasseau, D. (2001). C. R. Acad. Sci., Ser. IIc: Chim, 4, 161-171.

Gupta, K. C. \& Sutar, A. K. (2008). Coord. Chem. Rev. 252, 1420-1450.

Létard, J.-F., Guionneau, P., Codjovi, E., Lavastre, O., Bravic, D., Chasseau, D. \& Kahn, O. (1997). J. Am. Chem. Soc. 119, 10861-10862.

Létard, J.-F., Guionneau, P., Rabardel, L., Howard, J. A. K., Goeta, A. E., Chasseau, D. \& Kahn, O. (1998). Inorg. Chem. 37, 4432-4441.

Liu, Y., Xuan, W. \& Cui, Y. (2010). Adv. Mater. 22, 4112-4135.

Murray, K. S. (2008). Eur. J. Inorg. Chem. pp. 3101-3121.

Sheldrick, G. M. (2008). Acta Cryst. A64, 112-122.

Westrip, S. P. (2010). J. Appl. Cryst. 43, 920-925. 


\section{supporting information}

Acta Cryst. (2014). E70, o1094-o1095 [doi:10.1107/S160053681401959X]

\section{Crystal structure of (pyridin-2-ylmethylidene)(triphenylmethyl)amine}

\section{Chatphorn Theppitak, Manlika Meesangkaew, Songwuit Chanthee, Nimit Sriprang and Kittipong Chainok}

\section{S1. Comment}

Schiff bases and their complexes have received intense attention owing to their structural and functional diversities (Gupta \& Sutar, 2008; Liu et al., 2010). Much effort has been devoted in recent years to the design and synthesize of Schiff bases and their iron(II/III) complexes displaying spin-crossover (SCO) properties with the aim of developing a new generation of memory devices, multifunctional materials, and other magnetic applications (Murray, 2008; Goodwin, 2004 and references therein). The 2-pyridylmethanimine Schiff base derivative has been used as a chelating ligand in mononuclear iron(II) SCO complexes as they often possess field strengths that lie within the right region to facilitate temperature-mediated switching between the high spin (HS) and low spin (LS) states of the iron(II) centers (Guionneau et al., 2001; Capes et al., 2000; Létard et al., 1998; Létard et al., 1997). These ligands are also able to form non-covalent interactions such as hydrogen bonding and weaker electrostatic contracts, which will help to provide diversity to the architectures of structures, and can be associated with subtle effects on the molecular magnetic properties of compounds. For example, the $\mathrm{N}-2$-pyridylmethylene-4-(phenylethynyl)aniline (PM-PEA) was employed with [Fe(NCS) ${ }_{2}$ precursor complex to form the mononuclear SCO derivative, $\left[\mathrm{Fe}(\mathrm{PM}-\mathrm{PEA})_{2}(\mathrm{NCS})_{2}\right]$, exhibiting a thermal hysteresis loop of $37 \mathrm{~K}$ (Létard et al., 1997). The crystal structure has been studied at room temperature (HS state) and at $140 \mathrm{~K}$ (LS state) suggests that the cooperativity may be attributed to intermolecular $\pi-\pi$ stacking between phenyl rings and crucially the strength of the $\mathrm{C}-\mathrm{H} \cdots \mathrm{S}$ interactions. Following the above, a family of iron(II) compounds containing the 2-pyridylmethanimine Schiff base derivatives have been prepared and found to show different SCO behaviors that range from very smooth and incomplete for $\left[\mathrm{Fe}(\mathrm{PM}-\mathrm{TeA})_{2}(\mathrm{NCS})_{2}\right], \mathrm{PM}-\mathrm{TeA}=N-\left(2^{\prime}-\right.$ pyridylmethyl)-4-aminoterphenyl (Guionneau $e t$ al., 2001), smooth with almost no hysteresis for $\left[\mathrm{Fe}(\mathrm{PM}-\mathrm{AzA})_{2}(\mathrm{NCS})_{2}\right]$ and exceptionally abrupt for $[\mathrm{Fe}(\mathrm{PM}-$ $\left.\mathrm{BiA})_{2}(\mathrm{NCS})_{2}\right], \mathrm{PM}-\mathrm{AzA}=\mathrm{N}-\left(2^{\prime}-\right.$ pyridylmethyl $)-4-($ phenylazo $)$ aniline, $\mathrm{PM}-\mathrm{BiA}=\mathrm{N}$-(2'-pyridylmethyl $)-4$-aminobiphenyl (Capes et al., 2000). Our interest in the mononuclear iron(II) SCO complexes has led us to prepare a new $N$ bidentate Schiff base derivative (I), which has the triphenylmethane group attached to the 2-pyridylmethanimine moiety. It is anticipated that weak non-covalent forces such as $\mathrm{C}-\mathrm{H} \cdots \pi$ and $\pi-\pi$ interactions will help to stabilize the assembly as well as increase the dimensionality of the structure and may also serve as weak exchange pathways between the magnetic metal center. Herein we report the supramolecular structure of (I), which is mediated by both intra- and intermolecular ef $\mathrm{C}-\mathrm{H} \cdots \pi$ interactions.

The $N$-bidentate Schiff base derivative (I) can be obtained by condensation of 2-pyridinecarboxaldehyde with triphenylmethanamine. Single crystal $X$-ray diffraction analysis reveals that (I) crystallizes in the chiral orthorhombic space group $P 2_{1} 2_{1} 2_{1}$, with two molecules (denoted $A$ and $B$ ) representing the asymmetric unit as shown in Fig. 1 . The bond distances within the phenyl (rings 2-4 and 6-8) and pyridine (rings 1 and 5) rings are in the range 1.341 (4) - 1.402 (4) $\AA$, mean $1.381 \AA$, for molecule $A$, and 1.347 (4) - 1.400 (5) $\AA$, mean $1.381 \AA$, for molecule $B$. The $\mathrm{C}-\mathrm{N}$ distances in the 


\section{supporting information}

imine bonds are 1.268 (4) and 1.273 (4) $\AA$ for the $\mathrm{C} 6-\mathrm{N} 2$ and $\mathrm{C} 6{ }^{\prime}-\mathrm{N} 2^{\prime}$, respectively, which are in agreement with $\mathrm{C}=\mathrm{N}$ double bond character for the imine group of Schiff bases type (Guionneau et al., 2001). The C7-N2 and the C7'-N2' distances between the imine and the triphenylmethane groups are 1.483 (4) and 1.473 (4) $\AA$, respectively. These are consistent with the values expected for $\mathrm{C}-\mathrm{N}$ single bonding (Guionneau et al., 2001). The bond angles $\mathrm{C} 5-\mathrm{C} 6-\mathrm{N} 2$ of $118.5(2)^{\circ}$ and the corresponding $\mathrm{C}^{\prime}-\mathrm{C} 6^{\prime}-\mathrm{N} 2^{\prime}$ of $117.9(2)^{\circ}$ are closed to the ideal value of $120^{\circ}$. This fact is further confirmed its $s p^{2}$ character.

The molecules $A$ and $B$ adopt different conformations (the torsion angles $\mathrm{C} 6-\mathrm{N} 2-\mathrm{C} 7-\mathrm{C} 8=138.6(3)^{\circ}$ and $\mathrm{C} 6^{\prime}-\mathrm{N} 2^{\prime}$ $\left.-\mathrm{C} 7^{\prime}-\mathrm{C} 8^{\prime}=-137.1(2)^{\circ}\right)$ in the crystal of (I) and are linked in inversion-symmetric pairs by edge-to-face (ef) $\mathrm{C}-\mathrm{H}^{\cdots} \cdots \pi$ type to form an $A-B$ dimer. The imine group in both molecules $A$ and $B$ is approximately coplanar with the pyridine rings, with the $\mathrm{N} 1-\mathrm{C} 5-\mathrm{C} 6-\mathrm{N} 2$ and $\mathrm{N} 1{ }^{\prime}-\mathrm{C}^{\prime}-\mathrm{C}^{\prime}-\mathrm{N} 2^{\prime}$ torsion angles of 170.1 (3) and -172.0 (3) $\AA$, respectively. In molecule $A$, the mean plane of the 2 -pyridylmethanimine (ring $1-\mathrm{C} 6=\mathrm{N} 2$ ) moiety and atom $\mathrm{C} 7$ [maximum deviation $=$ 0.101 (2) $\AA$ for atom N2] is inclined to the phenyl rings 2, 3, and 4 by 47.2 (1), 84.7 (1), and 59.8 (1) ${ }^{\circ}$, respectively. Similarly, the dihedral angles between the mean plane of the corresponding ring $5-\mathrm{C} 6^{\prime}=\mathrm{N} 2^{\prime}-\mathrm{C} 7^{\prime}$ [maximum deviation $=0.054$ (2) $\AA$ for atom $\left.C 6^{\prime}\right]$ and the phenyl rings 6, 7, and 8 in the molecule $B$ are 47.5 (1), 83.9 (1), and $59.4(1)^{\circ}$, respectively. One of the interesting features of the crystal structure of (I) is the angle between the $\mathrm{C}=\mathrm{N}$ bond and the rings of the triphenylmethane group. Namely, the angles of $\mathrm{N} 2-\mathrm{C} 7-\mathrm{C} 20\left(116.3(2)^{\circ}\right)$ and $\mathrm{N} 2^{\prime}-\mathrm{C} 7^{\prime}-\mathrm{C} 20^{\prime}\left(116.5(2)^{\circ}\right)$ are much larger than the corresponding angles of $\mathrm{N} 2-\mathrm{C} 7-\mathrm{C} 8\left(106.4(2)^{\circ}\right), \mathrm{N} 2^{\prime}-\mathrm{C}^{\prime}-\mathrm{C} 8^{\prime}\left(106.2(2)^{\circ}\right), \mathrm{N} 2-\mathrm{C} 7-\mathrm{C} 14$ $\left(104.3(2)^{\circ}\right)$, and $\mathrm{N} 2^{\prime}-\mathrm{C}^{\prime}-\mathrm{C} 14^{\prime}\left(104.2(2)^{\circ}\right)$. This is presumably due to the inversion related intermolecular ef $\mathrm{C}-\mathrm{H} \cdots \pi$ type in the dimer which lock the molecular conformations.

Several intra- and intermolecular ef $\mathrm{C}-\mathrm{H} \cdots \pi$ interactions are observed in the crystal structure of (I) and have profound effects on both the molecular and the packing conformations. In the $A-B$ dimer, hydrogen atoms, $\mathrm{H} 1$ and $\mathrm{H} 1$ ' of the pyridine rings (rings 1 and 5) are point toward the centroid $(C g)$ of the phenyl rings (4 and 8) at distance of $2.819 \AA(\mathrm{C} 1$ $\left.-\mathrm{H} 1 \cdots C g 8=136.9^{\circ}\right)$ for the $\mathrm{H} 1 \cdots C g 8$ and $2.789 \AA\left(C 1^{\prime}-\mathrm{H} 1^{\prime} \cdots C g 4=137.1^{\circ}\right)$ for the $\mathrm{H} 1^{\prime} \cdots C g 1$. The 2-pyridylmethanimine moiety of molecules $A$ and $B$ is almost parallel with the dihedral angle of $0.6(1)^{\circ}$. It should be noted that the value of $\mathrm{C} \cdots \mathrm{N}$ contact $\left(\mathrm{C} 6 \cdots \mathrm{N} 1^{\prime}=3.943\right.$ (4) $\AA, \mathrm{C} 6-\mathrm{H} 6 \cdots \mathrm{N} 1^{\prime}=144.6^{\circ} ; \mathrm{C}^{\prime} \cdots \mathrm{N} 1=3.929$ (4) $\AA, \mathrm{C}^{\prime}-\mathrm{H} 6^{\prime} \cdots \mathrm{N} 1=$ $\left.144.3^{\circ}\right)$ is greatly longer than the sum of the van der Waals radii $[1.70(\mathrm{C})+1.54(\mathrm{~N})=3.24 \AA]$ (Bondi, 1964). Thus, no classical $\mathrm{C}-\mathrm{H} \cdots \mathrm{N}$ hydrogen bonds involving the imine and the pyridine groups are observed in the dimer. There are, however, additional intramolecular $\mathrm{C}-\mathrm{H} \cdots \pi$ hydrogen bonding between the adjacent phenyl rings (rings $2-4$ and 6-8) with the $\mathrm{H}^{\cdots} \cdot \mathrm{Cg}$ distances and the $\mathrm{C}-\mathrm{H} \cdots \mathrm{Cg}$ angle in the range $2.897-3.104 \AA$ and $119.8-133.3^{\circ}$, Table 1 . As shown in Fig. 2, the dimers are extended into a three-dimensional supramolecular architecture via the intermolecular ef $\mathrm{C}-\mathrm{H} \cdots \pi$ interactions with the $\mathrm{H} \cdots C g$ distance and the $\mathrm{C}-\mathrm{H} \cdots C g$ angle in the range $2.769-3.169 \AA$ and $116.0-145.1^{\circ}$, respectively (Table 1). Finally, no classical hydrogen bonding and $\pi-\pi$ stacking between adjacent molecules are observed in the crystal structure of (I). Its packing is mainly based on weak ef $\mathrm{C}-\mathrm{H} \cdots \pi$ interactions.

\section{S2. Experimental}

To a solution of 2-pyridinecarboxaldehyde $(1.90 \mathrm{ml}, 0.02 \mathrm{~mol})$ in benzene $(100 \mathrm{ml})$, and a few drops of acetic acid as catalyst was added drop wise with triphenylmethanamine $(5.20 \mathrm{~g}, 0.02 \mathrm{~mol})$ at room temperature. The reaction mixture was stirred under reflux at $110^{\circ} \mathrm{C}$. After $6 \mathrm{~h}$ of refluxing, the yellow solution was neutralized with $\mathrm{Na}_{2} \mathrm{CO}_{3}(2 \mathrm{mmol})$, filtered, and concentrated to dryness in vacuo. The residue was recrystallized from a mixture of $\mathrm{CH}_{2} \mathrm{Cl}_{2}$ and petroleum ether $(2: 1, v: v)$ to give white crystalline solid of (I). Anal. Found (calcd) for $\mathrm{C}_{25} \mathrm{H}_{20} \mathrm{~N}_{2}$ (348.16): $\mathrm{C}, 86.15$ (86.17); $\mathrm{H}, 5.81$ (5.79); $\mathrm{N}, 8.05$ (8.04). 


\section{S3. Refinement}

The $\mathrm{C}$-bound hydrogen atoms were placed in geometrically idealized positions based on chemical coordinations and constrained to ride on their parent atom positions with a $\mathrm{C}-\mathrm{H}$ distances of $0.95 \AA$ and with $U_{\text {iso }}(\mathrm{H})=1.2 U_{\text {eq }}(\mathrm{C})$ for the aromatic $\mathrm{H}$ atoms.

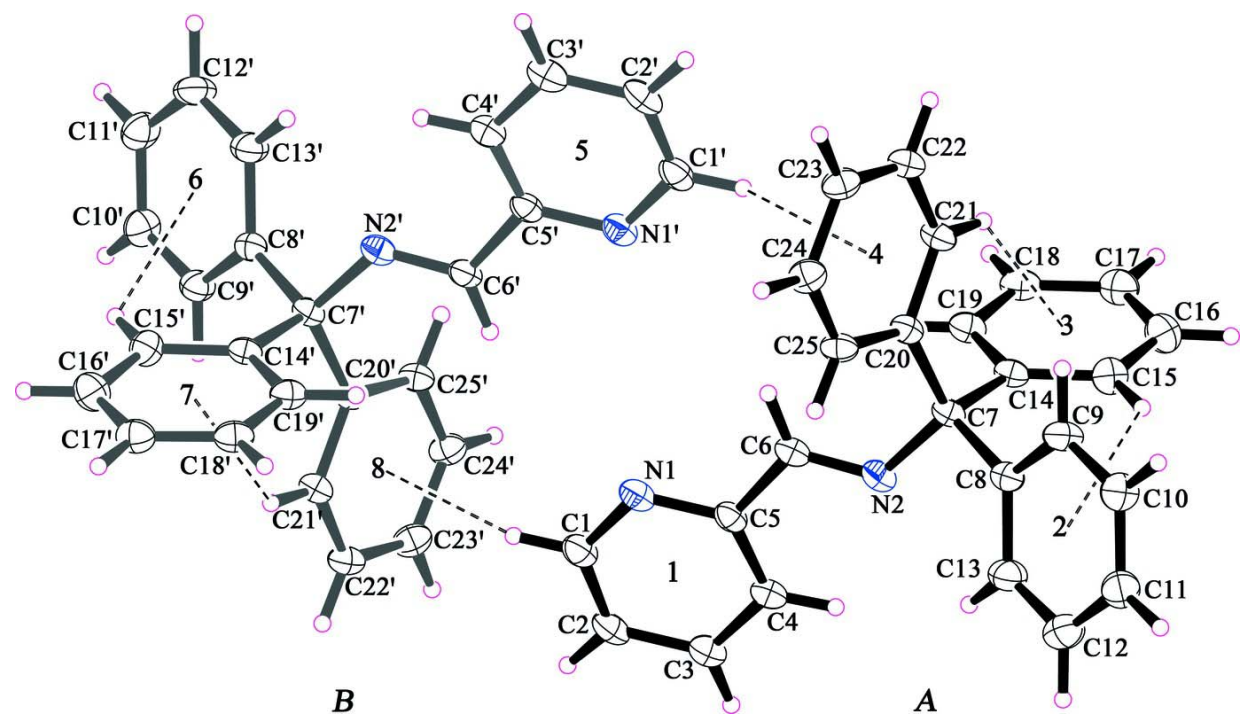

\section{Figure 1}

A view of the two symmetry-independent molecules $(A$ and $B)$ in (I), showing the atom-numbering scheme. Displacement ellipsoids are drawn at the $30 \%$ probability level. Covalent bonds in $A$ and $B$ molecules are shaded differently. The labeling scheme, $1-8$, applied to the aromatic rings are used to identify the rings in the subsequent discussion. 


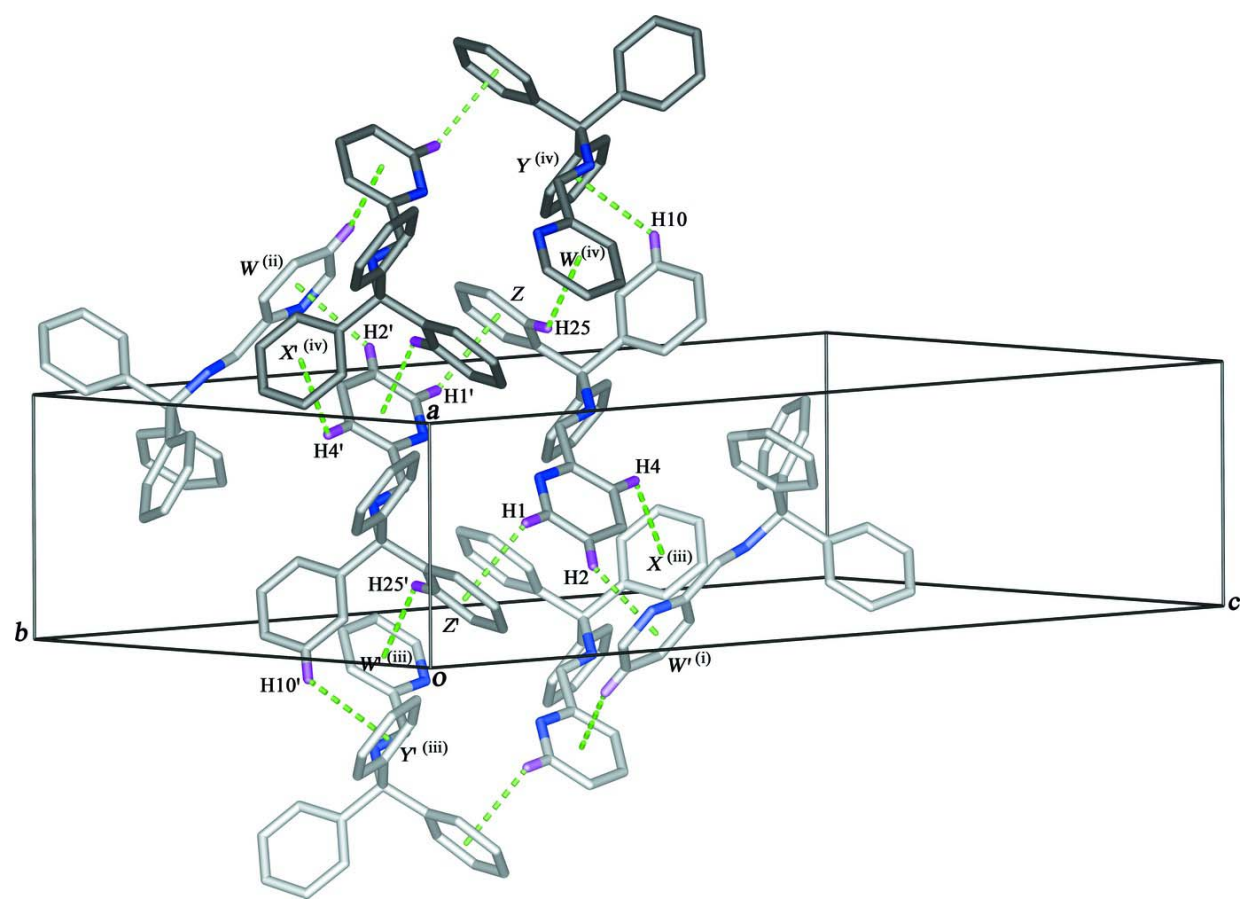

\section{Figure 2}

A packing diagram for (I), displaying the intermolecular ef $\mathrm{C}-\mathrm{H} \cdots \pi$ interactions (dashed lines). For clarity, only $\mathrm{H}$ atoms involved in $\mathrm{C}-\mathrm{H} \cdots \pi$ hydrogen bonding have been included. [Symmetry codes: (i) $1-\mathrm{x}, 1 / 2+y, 3 / 2-\mathrm{z}$; (ii) $-\mathrm{x}, y-1 / 2,3 / 2-$ z; (iii) $1+x, y, z$; (iv) $x-1, y, z]$.

\section{(Pyridin-2-ylmethylidene)(triphenylmethyl)amine}

\section{Crystal data}

$\mathrm{C}_{25} \mathrm{H}_{20} \mathrm{~N}_{2}$

$M_{r}=348.43$

Orthorhombic, $P 22_{1} 2_{1}$

Hall symbol: P 2ac 2ab

$a=7.0719(5) \AA$

$b=16.3972(8) \AA$

$c=31.880(2) \AA$

$V=3696.7(4) \AA^{3}$

$Z=8$

\section{Data collection}

Agilent Xcalibur (Sapphire3, Gemini ultra) diffractometer

Radiation source: fine-focus sealed tube

Mirror monochromator

Detector resolution: 8 pixels $\mathrm{mm}^{-1}$

$\omega$ scans

Absorption correction: multi-scan

(CrysAlis PRO and CrysAlis RED; Agilent, 2012)
$F(000)=1472$

$D_{\mathrm{x}}=1.252 \mathrm{Mg} \mathrm{m}^{-3}$

$\mathrm{Cu} K \alpha$ radiation, $\lambda=1.54178 \AA$

Cell parameters from 3448 reflections

$\mu=0.56 \mathrm{~mm}^{-1}$

$T=173 \mathrm{~K}$

Block, yellow

$0.30 \times 0.26 \times 0.22 \mathrm{~mm}$

$T_{\min }=0.849, T_{\max }=0.886$

21735 measured reflections

6965 independent reflections

5059 reflections with $I>2 \sigma(I)$

$R_{\text {int }}=0.058$

$\theta_{\text {max }}=73.1^{\circ}, \theta_{\text {min }}=9.8^{\circ}$

$h=-8 \rightarrow 8$

$k=-19 \rightarrow 15$

$l=-28 \rightarrow 39$ 


\section{Refinement}

Refinement on $F^{2}$

Least-squares matrix: full

$R\left[F^{2}>2 \sigma\left(F^{2}\right)\right]=0.057$

$w R\left(F^{2}\right)=0.153$

$S=1.00$

6965 reflections

487 parameters

0 restraints

Primary atom site location: structure-invariant direct methods

Secondary atom site location: difference Fourier map

\section{Special details}

Geometry. All e.s.d.'s (except the e.s.d. in the dihedral angle between two 1.s. planes) are estimated using the full covariance matrix. The cell e.s.d.'s are taken into account individually in the estimation of e.s.d.'s in distances, angles and torsion angles; correlations between e.s.d.'s in cell parameters are only used when they are defined by crystal symmetry. An approximate (isotropic) treatment of cell e.s.d.'s is used for estimating e.s.d.'s involving 1.s. planes.

Refinement. Refinement of $F^{2}$ against ALL reflections. The weighted $R$-factor $w R$ and goodness of fit $S$ are based on $F^{2}$, conventional $R$-factors $R$ are based on $F$, with $F$ set to zero for negative $F^{2}$. The threshold expression of $F^{2}>\sigma\left(F^{2}\right)$ is used only for calculating $R$-factors(gt) etc. and is not relevant to the choice of reflections for refinement. $R$-factors based on $F^{2}$ are statistically about twice as large as those based on $F$, and $R$ - factors based on ALL data will be even larger.

Fractional atomic coordinates and isotropic or equivalent isotropic displacement parameters $\left(\AA^{2}\right)$

\begin{tabular}{lllll}
\hline & $x$ & $y$ & $z$ & $U_{\text {iss }} / U_{\text {eq }}$ \\
\hline $\mathrm{N} 1$ & $0.3234(4)$ & $0.72675(16)$ & $0.72533(8)$ & $0.0395(6)$ \\
$\mathrm{N} 2$ & $0.0763(4)$ & $0.67303(16)$ & $0.63297(8)$ & $0.0368(6)$ \\
$\mathrm{C} 1$ & $0.4649(5)$ & $0.7751(2)$ & $0.73827(11)$ & $0.0408(7)$ \\
$\mathrm{H} 1$ & 0.4933 & 0.7761 & 0.7674 & $0.049^{*}$ \\
$\mathrm{C} 2$ & $0.5717(5)$ & $0.8232(2)$ & $0.71190(11)$ & $0.0419(8)$ \\
$\mathrm{H} 2$ & 0.6713 & 0.8561 & 0.7226 & $0.050^{*}$ \\
$\mathrm{C} 3$ & $0.5300(5)$ & $0.8225(2)$ & $0.66932(10)$ & $0.0419(8)$ \\
$\mathrm{H} 3$ & 0.5988 & 0.8559 & 0.6504 & $0.050^{*}$ \\
$\mathrm{C} 4$ & $0.3861(5)$ & $0.77209(19)$ & $0.65489(10)$ & $0.0385(7)$ \\
$\mathrm{H} 4$ & 0.3559 & 0.7696 & 0.6259 & $0.046^{*}$ \\
$\mathrm{C} 5$ & $0.2876(4)$ & $0.72545(18)$ & $0.68395(9)$ & $0.0339(7)$ \\
$\mathrm{C} 6$ & $0.1354(5)$ & $0.66888(19)$ & $0.67048(10)$ & $0.0359(7)$ \\
$\mathrm{H} 6$ & 0.0833 & 0.6304 & 0.6895 & $0.043^{*}$ \\
$\mathrm{C} 7$ & $-0.0641(4)$ & $0.61515(18)$ & $0.61548(9)$ & $0.0335(7)$ \\
$\mathrm{C} 8$ & $-0.2019(5)$ & $0.66622(19)$ & $0.58873(9)$ & $0.0355(7)$ \\
$\mathrm{C} 13$ & $-0.1588(5)$ & $0.7448(2)$ & $0.57610(11)$ & $0.0465(8)$ \\
$\mathrm{H} 13$ & -0.0432 & 0.7689 & 0.5848 & $0.056^{*}$ \\
$\mathrm{C} 12$ & $-0.2826(6)$ & $0.7891(2)$ & $0.55077(12)$ & $0.0560(10)$ \\
$\mathrm{H} 12$ & -0.2515 & 0.8432 & 0.5425 & $0.067^{*}$ \\
$\mathrm{C} 11$ & $-0.4510(6)$ & $0.7541(2)$ & $0.53771(11)$ & $0.0510(9)$ \\
$\mathrm{H} 11$ & -0.5344 & 0.7835 & 0.5199 & $0.061^{*}$ \\
C10 & $-0.4964(5)$ & $0.6768(2)$ & $0.55064(11)$ & $0.0470(9)$ \\
$\mathrm{H} 10$ & -0.6125 & 0.6529 & 0.5421 & $0.056^{*}$ \\
& & & &
\end{tabular}

Hydrogen site location: inferred from

$w=1 /\left[\sigma^{2}\left(F_{\mathrm{o}}^{2}\right)+(0.0771 P)^{2}\right]$

where $P=\left(F_{\mathrm{o}}^{2}+2 F_{\mathrm{c}}{ }^{2}\right) / 3$

$(\Delta / \sigma)_{\max }<0.001$

$\Delta \rho_{\min }=-0.18$ e $\AA^{-3}$

Absolute structure: Flack (1983), XXX Friedel

pairs

Absolute structure parameter: 0.3 (8) 


\begin{tabular}{|c|c|c|c|c|}
\hline $\mathrm{C} 9$ & $-0.3737(5)$ & $0.6334(2)$ & $0.57618(11)$ & $0.0432(8)$ \\
\hline H9 & -0.4079 & 0.5802 & 0.5852 & $0.052 *$ \\
\hline $\mathrm{C} 14$ & $0.0542(4)$ & $0.55700(18)$ & $0.58757(9)$ & $0.0338(7)$ \\
\hline $\mathrm{C} 15$ & $0.0086(5)$ & $0.5395(2)$ & $0.54651(10)$ & $0.0426(8)$ \\
\hline H15 & -0.0942 & 0.5670 & 0.5335 & $0.051^{*}$ \\
\hline $\mathrm{C} 16$ & $0.1108(6)$ & $0.4824(2)$ & $0.52396(11)$ & $0.0491(9)$ \\
\hline H16 & 0.0777 & 0.4712 & 0.4957 & $0.059^{*}$ \\
\hline $\mathrm{C} 17$ & $0.2588(5)$ & $0.4419(2)$ & $0.54197(11)$ & $0.0475(9)$ \\
\hline H17 & 0.3262 & 0.4019 & 0.5265 & $0.057^{*}$ \\
\hline $\mathrm{C} 18$ & $0.3102(5)$ & $0.4593(2)$ & $0.58270(10)$ & $0.0421(8)$ \\
\hline H18 & 0.4148 & 0.4322 & 0.5952 & $0.051^{*}$ \\
\hline C19 & $0.2088(5)$ & $0.51641(19)$ & $0.60531(10)$ & $0.0390(7)$ \\
\hline H19 & 0.2447 & 0.5283 & 0.6334 & $0.047^{*}$ \\
\hline $\mathrm{C} 20$ & $-0.1792(4)$ & $0.56558(19)$ & $0.64745(9)$ & $0.0330(6)$ \\
\hline $\mathrm{C} 21$ & $-0.2010(5)$ & $0.48179(19)$ & $0.64471(10)$ & $0.0373(7)$ \\
\hline $\mathrm{H} 21$ & -0.1317 & 0.4526 & 0.6241 & $0.045^{*}$ \\
\hline $\mathrm{C} 22$ & $-0.3221(5)$ & $0.4391(2)$ & $0.67150(10)$ & $0.0403(7)$ \\
\hline $\mathrm{H} 22$ & -0.3347 & 0.3817 & 0.6688 & $0.048 *$ \\
\hline $\mathrm{C} 23$ & $-0.4230(5)$ & $0.4802(2)$ & $0.70172(10)$ & $0.0407(7)$ \\
\hline $\mathrm{H} 23$ & -0.5065 & 0.4516 & 0.7198 & $0.049^{*}$ \\
\hline $\mathrm{C} 24$ & $-0.4017(5)$ & $0.5638(2)$ & $0.70542(10)$ & $0.0418(8)$ \\
\hline $\mathrm{H} 24$ & -0.4700 & 0.5925 & 0.7264 & $0.050^{*}$ \\
\hline $\mathrm{C} 25$ & $-0.2821(5)$ & 0.60567 (19) & $0.67889(10)$ & $0.0374(7)$ \\
\hline $\mathrm{H} 25$ & -0.2691 & 0.6630 & 0.6820 & $0.045^{*}$ \\
\hline $\mathrm{N} 1^{\prime}$ & $0.1678(4)$ & $0.52217(16)$ & $0.76822(8)$ & $0.0386(6)$ \\
\hline $\mathrm{C} 1^{\prime}$ & $0.0245(5)$ & $0.4739(2)$ & $0.75559(11)$ & $0.0412(8)$ \\
\hline $\mathrm{H} 1^{\prime}$ & -0.0053 & 0.4722 & 0.7266 & $0.049^{*}$ \\
\hline $\mathrm{N} 2^{\prime}$ & $0.4146(4)$ & $0.57659(16)$ & $0.86033(8)$ & $0.0363(6)$ \\
\hline $\mathrm{C} 2^{\prime}$ & $-0.0814(5)$ & $0.4267(2)$ & $0.78293(11)$ & $0.0447(8)$ \\
\hline $\mathrm{H} 2^{\prime}$ & -0.1825 & 0.3941 & 0.7728 & $0.054 *$ \\
\hline $\mathrm{C} 3^{\prime}$ & $-0.0381(5)$ & $0.4277(2)$ & $0.82482(11)$ & $0.0453(8)$ \\
\hline $\mathrm{H} 3^{\prime}$ & -0.1075 & 0.3952 & 0.8441 & $0.054 *$ \\
\hline $\mathrm{C} 4^{\prime}$ & $0.1078(5)$ & $0.4768(2)$ & $0.83851(10)$ & $0.0389(7)$ \\
\hline $\mathrm{H} 4^{\prime}$ & 0.1404 & 0.4789 & 0.8674 & $0.047^{*}$ \\
\hline $\mathrm{C} 5^{\prime}$ & $0.2058(4)$ & $0.52291(18)$ & $0.80935(9)$ & $0.0335(6)$ \\
\hline $\mathrm{C} 6^{\prime}$ & $0.3573(4)$ & $0.58008(19)$ & $0.82251(9)$ & $0.0348(7)$ \\
\hline H6 & 0.4100 & 0.6181 & 0.8033 & $0.042 *$ \\
\hline $\mathrm{C} 7^{\prime}$ & $0.5550(4)$ & $0.63331(18)$ & $0.87805(9)$ & $0.0315(6)$ \\
\hline $\mathrm{C} 8^{\prime}$ & $0.6910(5)$ & $0.58111(19)$ & $0.90443(9)$ & $0.0333(6)$ \\
\hline C9' & $0.8659(5)$ & $0.6119(2)$ & $0.91612(10)$ & $0.0400(7)$ \\
\hline H9' & 0.9025 & 0.6647 & 0.9069 & $0.048 *$ \\
\hline $\mathrm{C} 10^{\prime}$ & $0.9893(5)$ & $0.5664(2)$ & $0.94125(10)$ & $0.0448(8)$ \\
\hline $\mathrm{H} 10^{\prime}$ & 1.1085 & 0.5883 & 0.9490 & $0.054^{*}$ \\
\hline C11' & $0.9380(6)$ & $0.4904(2)$ & $0.95454(11)$ & $0.0506(9)$ \\
\hline H11' & 1.0206 & 0.4597 & 0.9720 & $0.061^{*}$ \\
\hline $\mathrm{C} 12^{\prime}$ & $0.7658(6)$ & $0.4580(2)$ & $0.94262(12)$ & $0.0507(9)$ \\
\hline $\mathrm{H} 12^{\prime}$ & 0.7315 & 0.4047 & 0.9515 & $0.061^{*}$ \\
\hline C13' & $0.6430(5)$ & $0.5026(2)$ & $0.91780(10)$ & $0.0438(8)$ \\
\hline
\end{tabular}




$\begin{array}{lllll}\mathrm{H} 13^{\prime} & 0.5251 & 0.4797 & 0.9098 & 0.053^{*} \\ \mathrm{C} 14^{\prime} & 0.4373(4) & 0.69107(18) & 0.90604(9) & 0.0330(7) \\ \mathrm{C} 15^{\prime} & 0.4871(5) & 0.7085(2) & 0.94706(10) & 0.0403(8) \\ \mathrm{H} 15^{\prime} & 0.5904 & 0.6807 & 0.9596 & 0.048^{*} \\ \mathrm{C} 16^{\prime} & 0.3873(6) & 0.7665(2) & 0.97014(11) & 0.0488(9) \\ \mathrm{H} 16^{\prime} & 0.4250 & 0.7788 & 0.9980 & 0.059^{*} \\ \mathrm{C} 17^{\prime} & 0.2338(5) & 0.8060(2) & 0.95266(10) & 0.0444(8) \\ \mathrm{H} 17^{\prime} & 0.1664 & 0.8458 & 0.9683 & 0.053^{*} \\ \mathrm{C} 18^{\prime} & 0.1797(5) & 0.7872(2) & 0.91230(10) & 0.0409(8) \\ \mathrm{H} 18^{\prime} & 0.0728 & 0.8133 & 0.9003 & 0.049^{*} \\ \mathrm{C} 19^{\prime} & 0.2796(4) & 0.73047(19) & 0.88923(10) & 0.0375(7) \\ \mathrm{H} 19^{\prime} & 0.2404 & 0.7181 & 0.8615 & 0.045^{*} \\ \mathrm{C} 20^{\prime} & 0.6711(4) & 0.68358(18) & 0.84642(9) & 0.0308(6) \\ \mathrm{C} 21^{\prime} & 0.6930(4) & 0.76739(19) & 0.84992(9) & 0.0348(7) \\ \mathrm{H} 21^{\prime} & 0.6231 & 0.7962 & 0.8706 & 0.042^{*} \\ \mathrm{C} 22^{\prime} & 0.8149(5) & 0.8098(2) & 0.82383(10) & 0.0392(7) \\ \mathrm{H} 22^{\prime} & 0.8284 & 0.8672 & 0.8268 & 0.047^{*} \\ \mathrm{C} 23^{\prime} & 0.9176(5) & 0.7686(2) & 0.79330(10) & 0.0423(8) \\ \mathrm{H} 23^{\prime} & 1.0034 & 0.7973 & 0.7758 & 0.051^{*} \\ \mathrm{C} 24^{\prime} & 0.8937(5) & 0.68586(19) & 0.78877(10) & 0.0388(7) \\ \mathrm{H} 24^{\prime} & 0.9604 & 0.6575 & 0.7674 & 0.047^{*} \\ \mathrm{C} 25^{\prime} & 0.7734(5) & 0.64361(19) & 0.81508(9) & 0.0361(7) \\ \text { H25'} & 0.7599 & 0.5863 & 0.8118 & 0.043^{*} \\ & & & & \end{array}$

Atomic displacement parameters $\left(\AA^{2}\right)$

\begin{tabular}{lllllll}
\hline & $U^{11}$ & $U^{22}$ & $U^{33}$ & $U^{12}$ & $U^{13}$ & $U^{23}$ \\
\hline $\mathrm{N} 1$ & $0.0380(14)$ & $0.0299(15)$ & $0.0505(15)$ & $-0.0005(12)$ & $-0.0043(12)$ & $-0.0053(12)$ \\
$\mathrm{N} 2$ & $0.0331(14)$ & $0.0311(14)$ & $0.0461(14)$ & $-0.0026(11)$ & $-0.0023(11)$ & $-0.0050(11)$ \\
$\mathrm{C} 1$ & $0.0409(17)$ & $0.0314(19)$ & $0.0502(18)$ & $0.0022(15)$ & $-0.0073(15)$ & $-0.0054(15)$ \\
$\mathrm{C} 2$ & $0.0355(17)$ & $0.0300(18)$ & $0.060(2)$ & $-0.0027(13)$ & $-0.0094(15)$ & $-0.0092(15)$ \\
$\mathrm{C} 3$ & $0.0403(18)$ & $0.0332(18)$ & $0.0521(18)$ & $-0.0052(14)$ & $-0.0007(15)$ & $-0.0021(14)$ \\
$\mathrm{C} 4$ & $0.0396(17)$ & $0.0265(17)$ & $0.0495(17)$ & $0.0003(14)$ & $0.0015(14)$ & $-0.0030(14)$ \\
$\mathrm{C} 5$ & $0.0333(15)$ & $0.0263(16)$ & $0.0420(16)$ & $0.0035(12)$ & $-0.0037(13)$ & $-0.0055(13)$ \\
$\mathrm{C} 6$ & $0.0359(16)$ & $0.0253(17)$ & $0.0465(17)$ & $-0.0004(13)$ & $0.0014(13)$ & $-0.0039(13)$ \\
$\mathrm{C} 7$ & $0.0322(14)$ & $0.0267(16)$ & $0.0415(16)$ & $-0.0058(12)$ & $-0.0007(13)$ & $-0.0021(12)$ \\
$\mathrm{C} 8$ & $0.0372(16)$ & $0.0290(17)$ & $0.0401(15)$ & $-0.0018(13)$ & $0.0027(13)$ & $-0.0010(13)$ \\
$\mathrm{C} 13$ & $0.049(2)$ & $0.037(2)$ & $0.0540(19)$ & $-0.0083(16)$ & $-0.0019(16)$ & $0.0041(15)$ \\
$\mathrm{C} 12$ & $0.065(3)$ & $0.045(2)$ & $0.059(2)$ & $0.002(2)$ & $-0.006(2)$ & $0.0096(17)$ \\
$\mathrm{C} 11$ & $0.056(2)$ & $0.043(2)$ & $0.053(2)$ & $0.0137(17)$ & $-0.0087(17)$ & $-0.0002(16)$ \\
$\mathrm{C} 10$ & $0.0377(18)$ & $0.050(2)$ & $0.0531(19)$ & $0.0015(16)$ & $-0.0034(15)$ & $0.0010(16)$ \\
$\mathrm{C} 9$ & $0.0402(18)$ & $0.0307(18)$ & $0.059(2)$ & $-0.0017(14)$ & $-0.0037(15)$ & $0.0065(15)$ \\
$\mathrm{C} 14$ & $0.0317(15)$ & $0.0266(16)$ & $0.0431(16)$ & $-0.0042(12)$ & $0.0025(13)$ & $0.0002(13)$ \\
$\mathrm{C} 15$ & $0.0359(17)$ & $0.047(2)$ & $0.0454(18)$ & $0.0007(16)$ & $-0.0005(14)$ & $-0.0005(15)$ \\
$\mathrm{C} 16$ & $0.050(2)$ & $0.049(2)$ & $0.0481(18)$ & $0.0021(18)$ & $0.0021(16)$ & $-0.0076(16)$ \\
$\mathrm{C} 17$ & $0.047(2)$ & $0.043(2)$ & $0.053(2)$ & $0.0017(17)$ & $0.0104(16)$ & $-0.0014(16)$ \\
$\mathrm{C} 18$ & $0.0352(17)$ & $0.0341(19)$ & $0.0571(19)$ & $-0.0004(14)$ & $0.0041(15)$ & $0.0052(14)$ \\
$\mathrm{C} 19$ & $0.0350(16)$ & $0.0318(18)$ & $0.0502(17)$ & $-0.0028(13)$ & $-0.0005(14)$ & $0.0003(14)$ \\
& & & & & &
\end{tabular}




\begin{tabular}{|c|c|c|c|c|c|c|}
\hline $\mathrm{C} 20$ & $0.0318(15)$ & $0.0264(16)$ & $0.0408(15)$ & $0.0008(12)$ & $-0.0018(12)$ & $-0.0020(12)$ \\
\hline $\mathrm{C} 21$ & $0.0386(17)$ & $0.0269(17)$ & $0.0464(17)$ & $-0.0060(14)$ & $0.0037(14)$ & $-0.0038(13)$ \\
\hline $\mathrm{C} 22$ & $0.0430(18)$ & $0.0280(17)$ & $0.0499(17)$ & $-0.0053(14)$ & $0.0003(15)$ & $0.0005(13)$ \\
\hline $\mathrm{C} 23$ & $0.0346(16)$ & $0.040(2)$ & $0.0474(17)$ & $-0.0012(14)$ & $0.0032(14)$ & $0.0097(14)$ \\
\hline $\mathrm{C} 24$ & $0.0387(17)$ & $0.040(2)$ & $0.0473(18)$ & $0.0079(15)$ & $0.0038(14)$ & $0.0039(14)$ \\
\hline $\mathrm{C} 25$ & $0.0379(16)$ & $0.0262(17)$ & $0.0481(17)$ & $0.0054(13)$ & $0.0046(14)$ & $0.0021(13)$ \\
\hline $\mathrm{N} 1^{\prime}$ & $0.0375(14)$ & $0.0302(15)$ & $0.0480(14)$ & $0.0020(11)$ & $-0.0039(11)$ & $-0.0060(12)$ \\
\hline $\mathrm{C} 1^{\prime}$ & $0.0427(17)$ & $0.0289(19)$ & $0.0519(18)$ & 0.0064 (14) & $-0.0076(14)$ & $-0.0104(14)$ \\
\hline $\mathrm{N} 2^{\prime}$ & $0.0331(13)$ & $0.0301(14)$ & $0.0456(14)$ & $-0.0055(11)$ & $-0.0010(11)$ & $-0.0025(11)$ \\
\hline $\mathrm{C} 2^{\prime}$ & $0.0357(17)$ & $0.0340(18)$ & $0.064(2)$ & $-0.0061(14)$ & $-0.0058(16)$ & $-0.0139(16)$ \\
\hline $\mathrm{C} 3^{\prime}$ & $0.0401(18)$ & $0.0351(19)$ & $0.061(2)$ & $-0.0049(14)$ & $0.0048(16)$ & $-0.0064(16)$ \\
\hline $\mathrm{C} 4^{\prime}$ & $0.0385(17)$ & $0.0319(18)$ & $0.0462(17)$ & $-0.0006(14)$ & $0.0031(14)$ & $-0.0048(14)$ \\
\hline $\mathrm{C} 5^{\prime}$ & $0.0335(15)$ & $0.0243(16)$ & $0.0428(15)$ & $0.0037(12)$ & $-0.0029(13)$ & $-0.0049(12)$ \\
\hline $\mathrm{C} 6^{\prime}$ & $0.0370(16)$ & $0.0239(17)$ & $0.0435(17)$ & $-0.0012(12)$ & $-0.0038(13)$ & $-0.0021(13)$ \\
\hline$C 7^{\prime}$ & $0.0290(14)$ & $0.0265(16)$ & $0.0388(15)$ & $-0.0028(12)$ & $-0.0010(12)$ & $-0.0025(12)$ \\
\hline $\mathrm{C} 8^{\prime}$ & $0.0365(15)$ & $0.0267(16)$ & $0.0368(15)$ & $0.0007(13)$ & $0.0003(12)$ & $-0.0002(12)$ \\
\hline $\mathrm{C} 9^{\prime}$ & $0.0385(17)$ & $0.0355(19)$ & $0.0459(18)$ & $-0.0047(14)$ & $-0.0047(14)$ & $0.0032(14)$ \\
\hline $\mathrm{C} 10^{\prime}$ & $0.0358(17)$ & $0.050(2)$ & $0.0484(18)$ & $0.0008(16)$ & $-0.0064(14)$ & $0.0027(16)$ \\
\hline $\mathrm{C} 11^{\prime}$ & $0.051(2)$ & $0.050(2)$ & $0.0513(19)$ & $0.0112(18)$ & $-0.0074(17)$ & $0.0075(17)$ \\
\hline $\mathrm{C} 12^{\prime}$ & $0.060(2)$ & $0.030(2)$ & $0.063(2)$ & $0.0001(17)$ & $-0.0034(19)$ & $0.0102(15)$ \\
\hline C13' & $0.046(2)$ & $0.0341(19)$ & $0.0510(18)$ & $-0.0077(15)$ & $-0.0027(15)$ & $0.0047(14)$ \\
\hline C14' & $0.0325(15)$ & $0.0267(16)$ & $0.0398(15)$ & $-0.0073(12)$ & $0.0017(12)$ & $0.0016(12)$ \\
\hline $\mathrm{C} 15^{\prime}$ & $0.0358(17)$ & $0.045(2)$ & $0.0399(16)$ & $0.0008(15)$ & $-0.0025(13)$ & $0.0014(14)$ \\
\hline $\mathrm{C} 16^{\prime}$ & $0.050(2)$ & $0.047(2)$ & $0.0494(19)$ & $0.0037(18)$ & $0.0028(16)$ & $-0.0078(16)$ \\
\hline $\mathrm{C} 17^{\prime}$ & $0.0445(19)$ & $0.038(2)$ & $0.0506(19)$ & $0.0042(16)$ & $0.0092(15)$ & $0.0007(15)$ \\
\hline $\mathrm{C} 18^{\prime}$ & $0.0340(16)$ & $0.035(2)$ & $0.0533(19)$ & $0.0007(14)$ & $0.0028(14)$ & $0.0058(14)$ \\
\hline C19' & $0.0332(15)$ & $0.0323(18)$ & $0.0469(17)$ & $-0.0017(13)$ & $-0.0008(13)$ & $0.0043(14)$ \\
\hline $\mathrm{C} 20^{\prime}$ & $0.0259(13)$ & $0.0249(16)$ & $0.0416(15)$ & $-0.0033(11)$ & $-0.0026(12)$ & $-0.0005(12)$ \\
\hline $\mathrm{C} 21^{\prime}$ & $0.0344(16)$ & $0.0277(17)$ & $0.0422(16)$ & $-0.0030(13)$ & $0.0005(13)$ & $-0.0034(13)$ \\
\hline $\mathrm{C} 22^{\prime}$ & $0.0396(17)$ & $0.0289(18)$ & $0.0492(17)$ & $-0.0049(14)$ & $-0.0004(15)$ & $0.0030(13)$ \\
\hline $\mathrm{C} 23^{\prime}$ & $0.0366(17)$ & $0.043(2)$ & $0.0472(18)$ & $0.0011(15)$ & $0.0050(14)$ & $0.0106(15)$ \\
\hline $\mathrm{C} 24^{\prime}$ & $0.0386(17)$ & $0.0349(19)$ & $0.0430(17)$ & $0.0078(14)$ & $0.0067(14)$ & $0.0047(14)$ \\
\hline $\mathrm{C} 25^{\prime}$ & $0.0387(16)$ & $0.0256(16)$ & $0.0440(16)$ & $0.0019(13)$ & $0.0019(13)$ & $-0.0004(13)$ \\
\hline
\end{tabular}

Geometric parameters (A, $\left.{ }^{o}\right)$

\begin{tabular}{llll}
\hline $\mathrm{N} 1-\mathrm{C} 1$ & $1.341(4)$ & $\mathrm{N} 1^{\prime}-\mathrm{C} 5^{\prime}$ & $1.339(4)$ \\
$\mathrm{N} 1-\mathrm{C} 5$ & $1.344(4)$ & $\mathrm{N} 1^{\prime}-\mathrm{C} 1^{\prime}$ & $1.347(4)$ \\
$\mathrm{N} 2-\mathrm{C} 6$ & $1.268(4)$ & $\mathrm{C} 1^{\prime}-\mathrm{C} 2^{\prime}$ & $1.385(5)$ \\
$\mathrm{N} 2-\mathrm{C} 7$ & $1.483(4)$ & $\mathrm{C} 1^{\prime}-\mathrm{H} 1^{\prime}$ & 0.9500 \\
$\mathrm{C} 1-\mathrm{C} 2$ & $1.379(5)$ & $\mathrm{N} 2^{\prime}-\mathrm{C} 6^{\prime}$ & $1.273(4)$ \\
$\mathrm{C} 1-\mathrm{H} 1$ & 0.9500 & $\mathrm{~N} 2^{\prime}-\mathrm{C} 7^{\prime}$ & $1.473(4)$ \\
$\mathrm{C} 2-\mathrm{C} 3$ & $1.389(5)$ & $\mathrm{C} 2^{\prime}-\mathrm{C} 3^{\prime}$ & $1.370(5)$ \\
$\mathrm{C} 2-\mathrm{H} 2$ & 0.9500 & $\mathrm{C} 3^{\prime}-\mathrm{C} 2^{\prime}$ & 0.9500 \\
$\mathrm{C} 3-\mathrm{C} 4$ & $1.389(5)$ & $\mathrm{C} 3^{\prime}-\mathrm{H} 3^{\prime}$ & $1.379(5)$ \\
$\mathrm{C} 3-\mathrm{H} 3$ & $\mathrm{C} 4^{\prime}-\mathrm{C} 5^{\prime}$ & 0.9500 \\
$\mathrm{C} 4-\mathrm{C} 5$ & 0.9500 & $\mathrm{C} 4^{\prime}-\mathrm{H} 4^{\prime}$ & $1.384(4)$ \\
$\mathrm{C} 4-\mathrm{H} 4$ & $1.388(4)$ & & 0.9500
\end{tabular}




\begin{tabular}{|c|c|c|c|}
\hline $\mathrm{C} 5-\mathrm{C} 6$ & $1.485(4)$ & $\mathrm{C} 5^{\prime}-\mathrm{C}^{\prime}$ & $1.484(4)$ \\
\hline $\mathrm{C} 6-\mathrm{H} 6$ & 0.9500 & $\mathrm{C} 6^{\prime}-\mathrm{H} 6^{\prime}$ & 0.9500 \\
\hline $\mathrm{C} 7-\mathrm{C} 20$ & $1.537(4)$ & $\mathrm{C} 7^{\prime}-\mathrm{C} 8^{\prime}$ & $1.538(4)$ \\
\hline $\mathrm{C} 7-\mathrm{C} 8$ & $1.542(4)$ & $\mathrm{C} 7^{\prime}-\mathrm{C} 20^{\prime}$ & $1.539(4)$ \\
\hline $\mathrm{C} 7-\mathrm{C} 14$ & $1.550(4)$ & $\mathrm{C} 7^{\prime}-\mathrm{C} 14^{\prime}$ & $1.545(4)$ \\
\hline $\mathrm{C} 8-\mathrm{C} 13$ & $1.384(4)$ & $\mathrm{C} 8^{\prime}-\mathrm{C} 9^{\prime}$ & $1.387(4)$ \\
\hline $\mathrm{C} 8-\mathrm{C} 9$ & $1.388(5)$ & $\mathrm{C} 8^{\prime}-\mathrm{C} 13^{\prime}$ & $1.397(4)$ \\
\hline $\mathrm{C} 13-\mathrm{C} 12$ & $1.395(5)$ & $\mathrm{C} 9^{\prime}-\mathrm{C} 10^{\prime}$ & $1.400(5)$ \\
\hline C13-H13 & 0.9500 & $\mathrm{C} 9^{\prime}-\mathrm{H} 9^{\prime}$ & 0.9500 \\
\hline $\mathrm{C} 12-\mathrm{C} 11$ & $1.386(6)$ & $\mathrm{C} 10^{\prime}-\mathrm{C} 11^{\prime}$ & $1.366(5)$ \\
\hline $\mathrm{C} 12-\mathrm{H} 12$ & 0.9500 & $\mathrm{C} 10^{\prime}-\mathrm{H} 10^{\prime}$ & 0.9500 \\
\hline $\mathrm{C} 11-\mathrm{C} 10$ & $1.371(5)$ & $\mathrm{C} 11^{\prime}-\mathrm{C} 12^{\prime}$ & $1.381(6)$ \\
\hline $\mathrm{C} 11-\mathrm{H} 11$ & 0.9500 & $\mathrm{C} 11^{\prime}-\mathrm{H} 11^{\prime}$ & 0.9500 \\
\hline $\mathrm{C} 10-\mathrm{C} 9$ & $1.387(5)$ & $\mathrm{C} 12^{\prime}-\mathrm{C} 13^{\prime}$ & $1.384(5)$ \\
\hline $\mathrm{C} 10-\mathrm{H} 10$ & 0.9500 & $\mathrm{C} 12^{\prime}-\mathrm{H} 12^{\prime}$ & 0.9500 \\
\hline $\mathrm{C} 9-\mathrm{H} 9$ & 0.9500 & $\mathrm{C} 13^{\prime}-\mathrm{H} 13^{\prime}$ & 0.9500 \\
\hline $\mathrm{C} 14-\mathrm{C} 15$ & $1.378(4)$ & $\mathrm{C} 14^{\prime}-\mathrm{C} 15^{\prime}$ & $1.384(4)$ \\
\hline $\mathrm{C} 14-\mathrm{C} 19$ & $1.399(4)$ & $\mathrm{C} 14^{\prime}-\mathrm{C} 19^{\prime}$ & $1.396(4)$ \\
\hline $\mathrm{C} 15-\mathrm{C} 16$ & $1.385(5)$ & $\mathrm{C} 15^{\prime}-\mathrm{C} 16^{\prime}$ & $1.393(5)$ \\
\hline C15-H15 & 0.9500 & $\mathrm{C} 15^{\prime}-\mathrm{H} 15^{\prime}$ & 0.9500 \\
\hline $\mathrm{C} 16-\mathrm{C} 17$ & $1.366(5)$ & $\mathrm{C} 16^{\prime}-\mathrm{C} 17^{\prime}$ & $1.382(5)$ \\
\hline $\mathrm{C} 16-\mathrm{H} 16$ & 0.9500 & $\mathrm{C} 16^{\prime}-\mathrm{H} 16^{\prime}$ & 0.9500 \\
\hline $\mathrm{C} 17-\mathrm{C} 18$ & $1.378(5)$ & $\mathrm{C} 17^{\prime}-\mathrm{C} 18^{\prime}$ & $1.378(5)$ \\
\hline C17-H17 & 0.9500 & $\mathrm{C} 17^{\prime}-\mathrm{H} 17^{\prime}$ & 0.9500 \\
\hline $\mathrm{C} 18-\mathrm{C} 19$ & $1.383(4)$ & $\mathrm{C} 18^{\prime}-\mathrm{C} 19^{\prime}$ & $1.380(4)$ \\
\hline C18-H18 & 0.9500 & $\mathrm{C} 18^{\prime}-\mathrm{H} 18^{\prime}$ & 0.9500 \\
\hline C19-H19 & 0.9500 & $\mathrm{C} 19^{\prime}-\mathrm{H} 19^{\prime}$ & 0.9500 \\
\hline $\mathrm{C} 20-\mathrm{C} 21$ & $1.385(4)$ & $\mathrm{C} 20^{\prime}-\mathrm{C} 21^{\prime}$ & $1.388(4)$ \\
\hline $\mathrm{C} 20-\mathrm{C} 25$ & $1.402(4)$ & $\mathrm{C} 20^{\prime}-\mathrm{C} 25^{\prime}$ & $1.397(4)$ \\
\hline $\mathrm{C} 21-\mathrm{C} 22$ & $1.397(4)$ & $\mathrm{C} 21^{\prime}-\mathrm{C} 22^{\prime}$ & $1.385(4)$ \\
\hline $\mathrm{C} 21-\mathrm{H} 21$ & 0.9500 & $\mathrm{C} 21^{\prime}-\mathrm{H} 21^{\prime}$ & 0.9500 \\
\hline $\mathrm{C} 22-\mathrm{C} 23$ & $1.375(5)$ & $\mathrm{C} 22^{\prime}-\mathrm{C} 23^{\prime}$ & $1.390(5)$ \\
\hline $\mathrm{C} 22-\mathrm{H} 22$ & 0.9500 & $\mathrm{C} 22^{\prime}-\mathrm{H} 22^{\prime}$ & 0.9500 \\
\hline $\mathrm{C} 23-\mathrm{C} 24$ & $1.383(5)$ & $\mathrm{C} 23^{\prime}-\mathrm{C} 24^{\prime}$ & $1.375(5)$ \\
\hline $\mathrm{C} 23-\mathrm{H} 23$ & 0.9500 & $\mathrm{C} 23^{\prime}-\mathrm{H} 23^{\prime}$ & 0.9500 \\
\hline $\mathrm{C} 24-\mathrm{C} 25$ & $1.379(5)$ & $\mathrm{C} 24^{\prime}-\mathrm{C} 25^{\prime}$ & $1.381(4)$ \\
\hline $\mathrm{C} 24-\mathrm{H} 24$ & 0.9500 & $\mathrm{C} 24^{\prime}-\mathrm{H} 24^{\prime}$ & 0.9500 \\
\hline $\mathrm{C} 25-\mathrm{H} 25$ & 0.9500 & $\mathrm{C} 25^{\prime}-\mathrm{H} 25^{\prime}$ & 0.9500 \\
\hline $\mathrm{C} 1-\mathrm{N} 1-\mathrm{C} 5$ & $116.9(3)$ & $\mathrm{C} 5^{\prime}-\mathrm{N} 1^{\prime}-\mathrm{C} 1^{\prime}$ & $116.7(3)$ \\
\hline $\mathrm{C} 6-\mathrm{N} 2-\mathrm{C} 7$ & $122.8(3)$ & $\mathrm{N} 1^{\prime}-\mathrm{C} 1^{\prime}-\mathrm{C} 2^{\prime}$ & $123.2(3)$ \\
\hline $\mathrm{N} 1-\mathrm{C} 1-\mathrm{C} 2$ & $124.0(3)$ & $\mathrm{N} 1^{\prime}-\mathrm{C} 1^{\prime}-\mathrm{H} 1^{\prime}$ & 118.4 \\
\hline $\mathrm{N} 1-\mathrm{C} 1-\mathrm{H} 1$ & 118.0 & $\mathrm{C} 2^{\prime}-\mathrm{C} 1^{\prime}-\mathrm{H} 1^{\prime}$ & 118.4 \\
\hline $\mathrm{C} 2-\mathrm{C} 1-\mathrm{H} 1$ & 118.0 & $\mathrm{C} 6^{\prime}-\mathrm{N} 2^{\prime}-\mathrm{C} 7^{\prime}$ & $123.3(3)$ \\
\hline $\mathrm{C} 1-\mathrm{C} 2-\mathrm{C} 3$ & $118.4(3)$ & $\mathrm{C} 3^{\prime}-\mathrm{C} 2^{\prime}-\mathrm{C} 1^{\prime}$ & $119.1(3)$ \\
\hline $\mathrm{C} 1-\mathrm{C} 2-\mathrm{H} 2$ & 120.8 & $\mathrm{C} 3^{\prime}-\mathrm{C} 2^{\prime}-\mathrm{H} 2^{\prime}$ & 120.5 \\
\hline $\mathrm{C} 3-\mathrm{C} 2-\mathrm{H} 2$ & 120.8 & $\mathrm{C} 1^{\prime}-\mathrm{C} 2^{\prime}-\mathrm{H} 2^{\prime}$ & 120.5 \\
\hline $\mathrm{C} 2-\mathrm{C} 3-\mathrm{C} 4$ & $119.0(3)$ & $\mathrm{C} 2^{\prime}-\mathrm{C} 3^{\prime}-\mathrm{C} 4^{\prime}$ & $118.8(3)$ \\
\hline
\end{tabular}




\begin{tabular}{|c|c|c|c|}
\hline $\mathrm{C} 2-\mathrm{C} 3-\mathrm{H} 3$ & 120.5 & $\mathrm{C} 2^{\prime}-\mathrm{C} 3^{\prime}-\mathrm{H} 3^{\prime}$ & 120.6 \\
\hline $\mathrm{C} 4-\mathrm{C} 3-\mathrm{H} 3$ & 120.5 & $\mathrm{C} 4^{\prime}-\mathrm{C} 3^{\prime}-\mathrm{H} 3^{\prime}$ & 120.6 \\
\hline $\mathrm{C} 5-\mathrm{C} 4-\mathrm{C} 3$ & $118.3(3)$ & $\mathrm{C} 3^{\prime}-\mathrm{C} 4^{\prime}-\mathrm{C} 5^{\prime}$ & $118.7(3)$ \\
\hline $\mathrm{C} 5-\mathrm{C} 4-\mathrm{H} 4$ & 120.8 & $\mathrm{C} 3^{\prime}-\mathrm{C} 4^{\prime}-\mathrm{H} 4^{\prime}$ & 120.6 \\
\hline $\mathrm{C} 3-\mathrm{C} 4-\mathrm{H} 4$ & 120.8 & $\mathrm{C} 5^{\prime}-\mathrm{C} 4^{\prime}-\mathrm{H} 4^{\prime}$ & 120.6 \\
\hline $\mathrm{N} 1-\mathrm{C} 5-\mathrm{C} 4$ & $123.5(3)$ & $\mathrm{N} 1^{\prime}-\mathrm{C} 5^{\prime}-\mathrm{C} 4^{\prime}$ & $123.5(3)$ \\
\hline $\mathrm{N} 1-\mathrm{C} 5-\mathrm{C} 6$ & $115.5(3)$ & $\mathrm{N} 1^{\prime}-\mathrm{C} 5^{\prime}-\mathrm{C} 6^{\prime}$ & $115.3(3)$ \\
\hline $\mathrm{C} 4-\mathrm{C} 5-\mathrm{C} 6$ & 121.0 & $\mathrm{C} 4^{\prime}-\mathrm{C} 5^{\prime}-\mathrm{C} 6^{\prime}$ & $121.1(3)$ \\
\hline $\mathrm{N} 2-\mathrm{C} 6-\mathrm{C} 5$ & $118.6(3)$ & $\mathrm{N} 2^{\prime}-\mathrm{C} 6^{\prime}-\mathrm{C} 5^{\prime}$ & $118.0(3)$ \\
\hline $\mathrm{N} 2-\mathrm{C} 6-\mathrm{H} 6$ & 120.7 & $\mathrm{~N} 2^{\prime}-\mathrm{C} 6^{\prime}-\mathrm{H} 6^{\prime}$ & 121.0 \\
\hline $\mathrm{C} 5-\mathrm{C} 6-\mathrm{H} 6$ & 120.7 & $\mathrm{C} 5^{\prime}-\mathrm{C} 6^{\prime}-\mathrm{H} 6^{\prime}$ & 121.0 \\
\hline $\mathrm{N} 2-\mathrm{C} 7-\mathrm{C} 20$ & $116.3(2)$ & $\mathrm{N} 2^{\prime}-\mathrm{C} 7^{\prime}-\mathrm{C} 8^{\prime}$ & $106.2(2)$ \\
\hline $\mathrm{N} 2-\mathrm{C} 7-\mathrm{C} 8$ & $106.5(2)$ & $\mathrm{N} 2^{\prime}-\mathrm{C} 7^{\prime}-\mathrm{C} 20^{\prime}$ & $116.5(2)$ \\
\hline $\mathrm{C} 20-\mathrm{C} 7-\mathrm{C} 8$ & $108.6(3)$ & $\mathrm{C} 8^{\prime}-\mathrm{C} 7^{\prime}-\mathrm{C} 20^{\prime}$ & $108.8(2)$ \\
\hline $\mathrm{N} 2-\mathrm{C} 7-\mathrm{C} 14$ & $104.4(2)$ & $\mathrm{N} 2^{\prime}-\mathrm{C} 7^{\prime}-\mathrm{C} 14^{\prime}$ & $104.2(2)$ \\
\hline $\mathrm{C} 20-\mathrm{C} 7-\mathrm{C} 14$ & $110.0(3)$ & $\mathrm{C} 8^{\prime}-\mathrm{C} 7^{\prime}-\mathrm{C} 14^{\prime}$ & $111.2(2)$ \\
\hline $\mathrm{C} 8-\mathrm{C} 7-\mathrm{C} 14$ & $111.0(2)$ & $\mathrm{C} 20^{\prime}-\mathrm{C} 7^{\prime}-\mathrm{C} 14^{\prime}$ & $109.7(2)$ \\
\hline $\mathrm{C} 13-\mathrm{C} 8-\mathrm{C} 9$ & $118.0(3)$ & $\mathrm{C} 9^{\prime}-\mathrm{C} 8^{\prime}-\mathrm{C} 13^{\prime}$ & $118.0(3)$ \\
\hline $\mathrm{C} 13-\mathrm{C} 8-\mathrm{C} 7$ & $121.9(3)$ & $\mathrm{C} 9^{\prime}-\mathrm{C} 8^{\prime}-\mathrm{C} 7^{\prime}$ & $120.1(3)$ \\
\hline $\mathrm{C} 9-\mathrm{C} 8-\mathrm{C} 7$ & $120.1(3)$ & $\mathrm{C} 13^{\prime}-\mathrm{C} 8^{\prime}-\mathrm{C} 7^{\prime}$ & $121.9(3)$ \\
\hline $\mathrm{C} 8-\mathrm{C} 13-\mathrm{C} 12$ & $121.0(4)$ & $\mathrm{C} 8^{\prime}-\mathrm{C} 9^{\prime}-\mathrm{C} 10^{\prime}$ & $121.0(3)$ \\
\hline $\mathrm{C} 8-\mathrm{C} 13-\mathrm{H} 13$ & 119.5 & $\mathrm{C} 8^{\prime}-\mathrm{C} 9^{\prime}-\mathrm{H} 9^{\prime}$ & 119.5 \\
\hline $\mathrm{C} 12-\mathrm{C} 13-\mathrm{H} 13$ & 119.5 & $\mathrm{C} 10^{\prime}-\mathrm{C} 9^{\prime}-\mathrm{H} 9^{\prime}$ & 119.5 \\
\hline $\mathrm{C} 11-\mathrm{C} 12-\mathrm{C} 13$ & $119.8(4)$ & $\mathrm{C} 11^{\prime}-\mathrm{C} 10^{\prime}-\mathrm{C} 9^{\prime}$ & $119.9(3)$ \\
\hline $\mathrm{C} 11-\mathrm{C} 12-\mathrm{H} 12$ & 120.1 & $\mathrm{C} 11^{\prime}-\mathrm{C} 10^{\prime}-\mathrm{H} 10^{\prime}$ & 120.1 \\
\hline $\mathrm{C} 13-\mathrm{C} 12-\mathrm{H} 12$ & 120.1 & $\mathrm{C} 9^{\prime}-\mathrm{C} 10^{\prime}-\mathrm{H} 10^{\prime}$ & 120.1 \\
\hline $\mathrm{C} 10-\mathrm{C} 11-\mathrm{C} 12$ & $119.6(4)$ & $\mathrm{C} 10^{\prime}-\mathrm{C} 11^{\prime}-\mathrm{C} 12^{\prime}$ & $120.0(3)$ \\
\hline $\mathrm{C} 10-\mathrm{C} 11-\mathrm{H} 11$ & 120.2 & $\mathrm{C} 10^{\prime}-\mathrm{C} 11^{\prime}-\mathrm{H} 11^{\prime}$ & 120.0 \\
\hline $\mathrm{C} 12-\mathrm{C} 11-\mathrm{H} 11$ & 120.2 & $\mathrm{C} 12^{\prime}-\mathrm{C} 11^{\prime}-\mathrm{H} 11^{\prime}$ & 120.0 \\
\hline $\mathrm{C} 11-\mathrm{C} 10-\mathrm{C} 9$ & $120.3(4)$ & $\mathrm{C} 11^{\prime}-\mathrm{C} 12^{\prime}-\mathrm{C} 13^{\prime}$ & $120.5(4)$ \\
\hline $\mathrm{C} 11-\mathrm{C} 10-\mathrm{H} 10$ & 119.8 & $\mathrm{C} 11^{\prime}-\mathrm{C} 12^{\prime}-\mathrm{H} 12^{\prime}$ & 119.8 \\
\hline $\mathrm{C} 9-\mathrm{C} 10-\mathrm{H} 10$ & 119.8 & $\mathrm{C} 13^{\prime}-\mathrm{C} 12^{\prime}-\mathrm{H} 12^{\prime}$ & 119.8 \\
\hline $\mathrm{C} 10-\mathrm{C} 9-\mathrm{C} 8$ & $121.2(3)$ & $\mathrm{C} 12^{\prime}-\mathrm{C} 13^{\prime}-\mathrm{C} 8^{\prime}$ & $120.6(3)$ \\
\hline $\mathrm{C} 10-\mathrm{C} 9-\mathrm{H} 9$ & 119.4 & $\mathrm{C} 12^{\prime}-\mathrm{C} 13^{\prime}-\mathrm{H} 13^{\prime}$ & 119.7 \\
\hline $\mathrm{C} 8-\mathrm{C} 9-\mathrm{H} 9$ & 119.4 & $\mathrm{C} 8^{\prime}-\mathrm{C} 13^{\prime}-\mathrm{H} 13^{\prime}$ & 119.7 \\
\hline $\mathrm{C} 15-\mathrm{C} 14-\mathrm{C} 19$ & $117.9(3)$ & $\mathrm{C} 15^{\prime}-\mathrm{C} 14^{\prime}-\mathrm{C} 19^{\prime}$ & $118.0(3)$ \\
\hline $\mathrm{C} 15-\mathrm{C} 14-\mathrm{C} 7$ & $123.1(3)$ & $\mathrm{C} 15^{\prime}-\mathrm{C} 14^{\prime}-\mathrm{C} 7^{\prime}$ & $122.4(3)$ \\
\hline $\mathrm{C} 19-\mathrm{C} 14-\mathrm{C} 7$ & $118.9(3)$ & $\mathrm{C} 19^{\prime}-\mathrm{C} 14^{\prime}-\mathrm{C} 7^{\prime}$ & $119.5(3)$ \\
\hline $\mathrm{C} 14-\mathrm{C} 15-\mathrm{C} 16$ & $120.8(3)$ & $\mathrm{C} 14^{\prime}-\mathrm{C} 15^{\prime}-\mathrm{C} 16^{\prime}$ & $120.7(3)$ \\
\hline $\mathrm{C} 14-\mathrm{C} 15-\mathrm{H} 15$ & 119.6 & $\mathrm{C} 14^{\prime}-\mathrm{C} 15^{\prime}-\mathrm{H} 15^{\prime}$ & 119.6 \\
\hline $\mathrm{C} 16-\mathrm{C} 15-\mathrm{H} 15$ & 119.6 & $\mathrm{C} 16^{\prime}-\mathrm{C} 15^{\prime}-\mathrm{H} 15^{\prime}$ & 119.6 \\
\hline $\mathrm{C} 17-\mathrm{C} 16-\mathrm{C} 15$ & $120.7(3)$ & $\mathrm{C} 17^{\prime}-\mathrm{C} 16^{\prime}-\mathrm{C} 15^{\prime}$ & $120.3(3)$ \\
\hline $\mathrm{C} 17-\mathrm{C} 16-\mathrm{H} 16$ & 119.7 & $\mathrm{C} 17^{\prime}-\mathrm{C} 16^{\prime}-\mathrm{H} 16^{\prime}$ & 119.8 \\
\hline $\mathrm{C} 15-\mathrm{C} 16-\mathrm{H} 16$ & 119.7 & $\mathrm{C} 15^{\prime}-\mathrm{C} 16^{\prime}-\mathrm{H} 16^{\prime}$ & 119.8 \\
\hline $\mathrm{C} 16-\mathrm{C} 17-\mathrm{C} 18$ & $119.9(3)$ & $\mathrm{C} 18^{\prime}-\mathrm{C} 17^{\prime}-\mathrm{C} 16^{\prime}$ & $119.3(3)$ \\
\hline $\mathrm{C} 16-\mathrm{C} 17-\mathrm{H} 17$ & 120.1 & $\mathrm{C} 18^{\prime}-\mathrm{C} 17^{\prime}-\mathrm{H} 17^{\prime}$ & 120.3 \\
\hline $\mathrm{C} 18-\mathrm{C} 17-\mathrm{H} 17$ & 120.1 & $\mathrm{C} 16^{\prime}-\mathrm{C} 17^{\prime}-\mathrm{H} 17^{\prime}$ & 120.3 \\
\hline $\mathrm{C} 17-\mathrm{C} 18-\mathrm{C} 19$ & $119.6(3)$ & $\mathrm{C} 17^{\prime}-\mathrm{C} 18^{\prime}-\mathrm{C} 19^{\prime}$ & $120.4(3)$ \\
\hline
\end{tabular}




\begin{tabular}{|c|c|c|c|}
\hline $\mathrm{C} 17-\mathrm{C} 18-\mathrm{H} 18$ & 120.2 & $\mathrm{C} 17^{\prime}-\mathrm{C} 18^{\prime}-\mathrm{H} 18^{\prime}$ & 119.8 \\
\hline $\mathrm{C} 19-\mathrm{C} 18-\mathrm{H} 18$ & 120.2 & $\mathrm{C} 19^{\prime}-\mathrm{C} 18^{\prime}-\mathrm{H} 18^{\prime}$ & 119.8 \\
\hline $\mathrm{C} 18-\mathrm{C} 19-\mathrm{C} 14$ & $121.1(3)$ & $\mathrm{C} 18^{\prime}-\mathrm{C} 19^{\prime}-\mathrm{C} 14^{\prime}$ & $121.1(3)$ \\
\hline $\mathrm{C} 18-\mathrm{C} 19-\mathrm{H} 19$ & 119.4 & $\mathrm{C} 18^{\prime}-\mathrm{C} 19^{\prime}-\mathrm{H} 19^{\prime}$ & 119.5 \\
\hline $\mathrm{C} 14-\mathrm{C} 19-\mathrm{H} 19$ & 119.4 & $\mathrm{C} 14^{\prime}-\mathrm{C} 19^{\prime}-\mathrm{H} 19^{\prime}$ & 119.5 \\
\hline $\mathrm{C} 21-\mathrm{C} 20-\mathrm{C} 25$ & $116.9(3)$ & $\mathrm{C} 21^{\prime}-\mathrm{C} 20^{\prime}-\mathrm{C} 25^{\prime}$ & $117.7(3)$ \\
\hline $\mathrm{C} 21-\mathrm{C} 20-\mathrm{C} 7$ & $122.8(3)$ & $\mathrm{C} 21^{\prime}-\mathrm{C} 20^{\prime}-\mathrm{C} 7^{\prime}$ & $122.5(3)$ \\
\hline $\mathrm{C} 25-\mathrm{C} 20-\mathrm{C} 7$ & $120.1(3)$ & $\mathrm{C} 25^{\prime}-\mathrm{C} 20^{\prime}-\mathrm{C} 7^{\prime}$ & $119.6(3)$ \\
\hline $\mathrm{C} 20-\mathrm{C} 21-\mathrm{C} 22$ & $121.7(3)$ & $\mathrm{C} 22^{\prime}-\mathrm{C} 21^{\prime}-\mathrm{C} 20^{\prime}$ & $121.3(3)$ \\
\hline $\mathrm{C} 20-\mathrm{C} 21-\mathrm{H} 21$ & 119.1 & $\mathrm{C} 22^{\prime}-\mathrm{C} 21^{\prime}-\mathrm{H} 21^{\prime}$ & 119.4 \\
\hline $\mathrm{C} 22-\mathrm{C} 21-\mathrm{H} 21$ & 119.1 & $\mathrm{C} 20^{\prime}-\mathrm{C} 21^{\prime}-\mathrm{H} 21^{\prime}$ & 119.4 \\
\hline $\mathrm{C} 23-\mathrm{C} 22-\mathrm{C} 21$ & $120.1(3)$ & $\mathrm{C} 21^{\prime}-\mathrm{C} 22^{\prime}-\mathrm{C} 23^{\prime}$ & $120.1(3)$ \\
\hline $\mathrm{C} 23-\mathrm{C} 22-\mathrm{H} 22$ & 120.0 & $\mathrm{C} 21^{\prime}-\mathrm{C} 22^{\prime}-\mathrm{H} 22^{\prime}$ & 120.0 \\
\hline $\mathrm{C} 21-\mathrm{C} 22-\mathrm{H} 22$ & 120.0 & $\mathrm{C} 23^{\prime}-\mathrm{C} 22^{\prime}-\mathrm{H} 22^{\prime}$ & 120.0 \\
\hline $\mathrm{C} 22-\mathrm{C} 23-\mathrm{C} 24$ & $119.2(3)$ & $\mathrm{C} 24^{\prime}-\mathrm{C} 23^{\prime}-\mathrm{C} 22^{\prime}$ & $119.3(3)$ \\
\hline $\mathrm{C} 22-\mathrm{C} 23-\mathrm{H} 23$ & 120.4 & $\mathrm{C} 24^{\prime}-\mathrm{C} 23^{\prime}-\mathrm{H} 23^{\prime}$ & 120.4 \\
\hline $\mathrm{C} 24-\mathrm{C} 23-\mathrm{H} 23$ & 120.4 & $\mathrm{C} 22^{\prime}-\mathrm{C} 23^{\prime}-\mathrm{H} 23^{\prime}$ & 120.4 \\
\hline $\mathrm{C} 25-\mathrm{C} 24-\mathrm{C} 23$ & $120.5(3)$ & $\mathrm{C} 23^{\prime}-\mathrm{C} 24^{\prime}-\mathrm{C} 25^{\prime}$ & $120.4(3)$ \\
\hline $\mathrm{C} 25-\mathrm{C} 24-\mathrm{H} 24$ & 119.7 & $\mathrm{C} 23^{\prime}-\mathrm{C} 24^{\prime}-\mathrm{H} 24^{\prime}$ & 119.8 \\
\hline $\mathrm{C} 23-\mathrm{C} 24-\mathrm{H} 24$ & 119.7 & $\mathrm{C} 25^{\prime}-\mathrm{C} 24^{\prime}-\mathrm{H} 24^{\prime}$ & 119.8 \\
\hline $\mathrm{C} 24-\mathrm{C} 25-\mathrm{C} 20$ & $121.5(3)$ & $\mathrm{C} 24^{\prime}-\mathrm{C} 25^{\prime}-\mathrm{C} 20^{\prime}$ & $121.2(3)$ \\
\hline $\mathrm{C} 24-\mathrm{C} 25-\mathrm{H} 25$ & 119.2 & $\mathrm{C} 24^{\prime}-\mathrm{C} 25^{\prime}-\mathrm{H} 25^{\prime}$ & 119.4 \\
\hline $\mathrm{C} 20-\mathrm{C} 25-\mathrm{H} 25$ & 119.2 & $\mathrm{C} 20^{\prime}-\mathrm{C} 25^{\prime}-\mathrm{H} 25^{\prime}$ & 119.4 \\
\hline $\mathrm{C} 5-\mathrm{N} 1-\mathrm{C} 1-\mathrm{C} 2$ & $0.9(5)$ & $\mathrm{C} 5^{\prime}-\mathrm{N} 1^{\prime}-\mathrm{C} 1^{\prime}-\mathrm{C} 2^{\prime}$ & $0.1(5)$ \\
\hline $\mathrm{N} 1-\mathrm{C} 1-\mathrm{C} 2-\mathrm{C} 3$ & $0.5(5)$ & $\mathrm{N} 1^{\prime}-\mathrm{C} 1^{\prime}-\mathrm{C} 2^{\prime}-\mathrm{C} 3^{\prime}$ & $-0.9(5)$ \\
\hline $\mathrm{C} 1-\mathrm{C} 2-\mathrm{C} 3-\mathrm{C} 4$ & $-1.5(5)$ & $\mathrm{C} 1^{\prime}-\mathrm{C} 2^{\prime}-\mathrm{C} 3^{\prime}-\mathrm{C} 4^{\prime}$ & $1.0(5)$ \\
\hline $\mathrm{C} 2-\mathrm{C} 3-\mathrm{C} 4-\mathrm{C} 5$ & $1.1(5)$ & $\mathrm{C} 2^{\prime}-\mathrm{C} 3^{\prime}-\mathrm{C} 4^{\prime}-\mathrm{C} 5^{\prime}$ & $-0.3(5)$ \\
\hline $\mathrm{C} 1-\mathrm{N} 1-\mathrm{C} 5-\mathrm{C} 4$ & $-1.3(5)$ & $\mathrm{C} 1^{\prime}-\mathrm{N} 1^{\prime}-\mathrm{C} 5^{\prime}-\mathrm{C} 4^{\prime}$ & $0.7(4)$ \\
\hline $\mathrm{C} 1-\mathrm{N} 1-\mathrm{C} 5-\mathrm{C} 6$ & $177.6(3)$ & $\mathrm{C} 1^{\prime}-\mathrm{N} 1^{\prime}-\mathrm{C} 5^{\prime}-\mathrm{C} 6^{\prime}$ & $-176.8(3)$ \\
\hline $\mathrm{C} 3-\mathrm{C} 4-\mathrm{C} 5-\mathrm{N} 1$ & $0.3(5)$ & $\mathrm{C} 3^{\prime}-\mathrm{C} 4^{\prime}-\mathrm{C} 5^{\prime}-\mathrm{N} 1^{\prime}$ & $-0.6(5)$ \\
\hline $\mathrm{C} 3-\mathrm{C} 4-\mathrm{C} 5-\mathrm{C} 6$ & $-178.5(3)$ & $\mathrm{C} 3^{\prime}-\mathrm{C} 4^{\prime}-\mathrm{C} 5^{\prime}-\mathrm{C} 6^{\prime}$ & $176.7(3)$ \\
\hline $\mathrm{C} 7-\mathrm{N} 2-\mathrm{C} 6-\mathrm{C} 5$ & $175.0(3)$ & $\mathrm{C} 7^{\prime}-\mathrm{N} 2^{\prime}-\mathrm{C} 6^{\prime}-\mathrm{C} 5^{\prime}$ & $-175.9(3)$ \\
\hline $\mathrm{N} 1-\mathrm{C} 5-\mathrm{C} 6-\mathrm{N} 2$ & $170.1(3)$ & $\mathrm{N} 1^{\prime}-\mathrm{C} 5^{\prime}-\mathrm{C} 6^{\prime}-\mathrm{N} 2^{\prime}$ & $-172.0(3)$ \\
\hline $\mathrm{C} 4-\mathrm{C} 5-\mathrm{C} 6-\mathrm{N} 2$ & $-10.9(5)$ & $\mathrm{C} 4^{\prime}-\mathrm{C} 5^{\prime}-\mathrm{C} 6^{\prime}-\mathrm{N} 2^{\prime}$ & $10.5(4)$ \\
\hline $\mathrm{C} 6-\mathrm{N} 2-\mathrm{C} 7-\mathrm{C} 20$ & $17.4(4)$ & $\mathrm{C} 6^{\prime}-\mathrm{N} 2^{\prime}-\mathrm{C} 7^{\prime}-\mathrm{C} 8^{\prime}$ & $-137.2(3)$ \\
\hline $\mathrm{C} 6-\mathrm{N} 2-\mathrm{C} 7-\mathrm{C} 8$ & $138.6(3)$ & $\mathrm{C} 6^{\prime}-\mathrm{N} 2^{\prime}-\mathrm{C} 7^{\prime}-\mathrm{C} 20^{\prime}$ & $-15.8(4)$ \\
\hline $\mathrm{C} 6-\mathrm{N} 2-\mathrm{C} 7-\mathrm{C} 14$ & $-103.9(3)$ & $\mathrm{C} 6^{\prime}-\mathrm{N} 2^{\prime}-\mathrm{C} 7^{\prime}-\mathrm{C} 14^{\prime}$ & $105.2(3)$ \\
\hline $\mathrm{N} 2-\mathrm{C} 7-\mathrm{C} 8-\mathrm{C} 13$ & $15.9(4)$ & $\mathrm{N} 2^{\prime}-\mathrm{C} 7^{\prime}-\mathrm{C} 8^{\prime}-\mathrm{C} 9^{\prime}$ & $162.9(3)$ \\
\hline $\mathrm{C} 20-\mathrm{C} 7-\mathrm{C} 8-\mathrm{C} 13$ & $141.9(3)$ & $\mathrm{C} 20^{\prime}-\mathrm{C} 7^{\prime}-\mathrm{C} 8^{\prime}-\mathrm{C} 9^{\prime}$ & 36.7 (4) \\
\hline $\mathrm{C} 14-\mathrm{C} 7-\mathrm{C} 8-\mathrm{C} 13$ & $-97.1(3)$ & $\mathrm{C} 14^{\prime}-\mathrm{C} 7^{\prime}-\mathrm{C} 8^{\prime}-\mathrm{C} 9^{\prime}$ & $-84.3(3)$ \\
\hline $\mathrm{N} 2-\mathrm{C} 7-\mathrm{C} 8-\mathrm{C} 9$ & $-164.9(3)$ & $\mathrm{N} 2^{\prime}-\mathrm{C} 7^{\prime}-\mathrm{C} 8^{\prime}-\mathrm{C} 13^{\prime}$ & $-18.0(4)$ \\
\hline $\mathrm{C} 20-\mathrm{C} 7-\mathrm{C} 8-\mathrm{C} 9$ & $-38.9(4)$ & $\mathrm{C} 20^{\prime}-\mathrm{C} 7^{\prime}-\mathrm{C} 8^{\prime}-\mathrm{C} 13^{\prime}$ & $-144.2(3)$ \\
\hline $\mathrm{C} 14-\mathrm{C} 7-\mathrm{C} 8-\mathrm{C} 9$ & $82.1(4)$ & $\mathrm{C} 14^{\prime}-\mathrm{C} 7^{\prime}-\mathrm{C} 8^{\prime}-\mathrm{C} 13^{\prime}$ & $94.8(3)$ \\
\hline $\mathrm{C} 9-\mathrm{C} 8-\mathrm{C} 13-\mathrm{C} 12$ & $-1.3(5)$ & $\mathrm{C} 13^{\prime}-\mathrm{C} 8^{\prime}-\mathrm{C} 9^{\prime}-\mathrm{C} 10^{\prime}$ & $-1.2(5)$ \\
\hline $\mathrm{C} 7-\mathrm{C} 8-\mathrm{C} 13-\mathrm{C} 12$ & $177.9(3)$ & $\mathrm{C} 7^{\prime}-\mathrm{C} 8^{\prime}-\mathrm{C} 9^{\prime}-\mathrm{C} 10^{\prime}$ & $178.0(3)$ \\
\hline $\mathrm{C} 8-\mathrm{C} 13-\mathrm{C} 12-\mathrm{C} 11$ & $-0.4(6)$ & $\mathrm{C} 8^{\prime}-\mathrm{C} 9^{\prime}-\mathrm{C} 10^{\prime}-\mathrm{C} 11^{\prime}$ & $0.1(5)$ \\
\hline $\mathrm{C} 13-\mathrm{C} 12-\mathrm{C} 11-\mathrm{C} 10$ & $1.5(6)$ & $\mathrm{C} 9^{\prime}-\mathrm{C} 10^{\prime}-\mathrm{C} 11^{\prime}-\mathrm{C} 12^{\prime}$ & $1.1(6)$ \\
\hline
\end{tabular}




\section{supporting information}

$\begin{array}{ll}\mathrm{C} 12-\mathrm{C} 11-\mathrm{C} 10-\mathrm{C} 9 & -0.9(6) \\ \mathrm{C} 11-\mathrm{C} 10-\mathrm{C} 9-\mathrm{C} 8 & -0.9(5) \\ \mathrm{C} 13-\mathrm{C} 8-\mathrm{C} 9-\mathrm{C} 10 & 2.0(5) \\ \mathrm{C} 7-\mathrm{C} 8-\mathrm{C} 9-\mathrm{C} 10 & -177.2(3) \\ \mathrm{N} 2-\mathrm{C} 7-\mathrm{C} 14-\mathrm{C} 15 & -129.1(3) \\ \mathrm{C} 20-\mathrm{C} 7-\mathrm{C} 14-\mathrm{C} 15 & 105.4(3) \\ \mathrm{C} 8-\mathrm{C} 7-\mathrm{C} 14-\mathrm{C} 15 & -14.8(4) \\ \mathrm{N} 2-\mathrm{C} 7-\mathrm{C} 14-\mathrm{C} 19 & 54.7(4) \\ \mathrm{C} 20-\mathrm{C} 7-\mathrm{C} 14-\mathrm{C} 19 & -70.8(3) \\ \mathrm{C} 8-\mathrm{C} 7-\mathrm{C} 14-\mathrm{C} 19 & 169.0(3) \\ \mathrm{C} 19-\mathrm{C} 14-\mathrm{C} 15-\mathrm{C} 16 & 1.2(5) \\ \mathrm{C} 7-\mathrm{C} 14-\mathrm{C} 15-\mathrm{C} 16 & -175.0(3) \\ \mathrm{C} 14-\mathrm{C} 15-\mathrm{C} 16-\mathrm{C} 17 & 0.2(6) \\ \mathrm{C} 15-\mathrm{C} 16-\mathrm{C} 17-\mathrm{C} 18 & -1.5(6) \\ \mathrm{C} 16-\mathrm{C} 17-\mathrm{C} 18-\mathrm{C} 19 & 1.4(5) \\ \mathrm{C} 17-\mathrm{C} 18-\mathrm{C} 19-\mathrm{C} 14 & 0.1(5) \\ \mathrm{C} 15-\mathrm{C} 14-\mathrm{C} 19-\mathrm{C} 18 & -1.4(5) \\ \mathrm{C} 7-\mathrm{C} 14-\mathrm{C} 19-\mathrm{C} 18 & 175.1(3) \\ \mathrm{N} 2-\mathrm{C} 7-\mathrm{C} 20-\mathrm{C} 21 & -130.8(3) \\ \mathrm{C} 8-\mathrm{C} 7-\mathrm{C} 20-\mathrm{C} 21 & 109.1(3) \\ \mathrm{C} 14-\mathrm{C} 7-\mathrm{C} 20-\mathrm{C} 21 & -12.5(4) \\ \mathrm{N} 2-\mathrm{C} 7-\mathrm{C} 20-\mathrm{C} 25 & 55.1(4) \\ \mathrm{C} 8-\mathrm{C} 7-\mathrm{C} 20-\mathrm{C} 25 & -64.9(4) \\ \mathrm{C} 14-\mathrm{C} 7-\mathrm{C} 20-\mathrm{C} 25 & 173.4(3) \\ \mathrm{C} 25-\mathrm{C} 20-\mathrm{C} 21-\mathrm{C} 22 & 1.1(5) \\ \mathrm{C} 7-\mathrm{C} 20-\mathrm{C} 21-\mathrm{C} 22 & -173.2(3) \\ \mathrm{C} 20-\mathrm{C} 21-\mathrm{C} 22-\mathrm{C} 23 & -0.3(5) \\ \mathrm{C} 21-\mathrm{C} 22-\mathrm{C} 23-\mathrm{C} 24 & -0.6(5) \\ \mathrm{C} 22-\mathrm{C} 23-\mathrm{C} 24-\mathrm{C} 25 & 0.6(5) \\ \mathrm{C} 23-\mathrm{C} 24-\mathrm{C} 25-\mathrm{C} 20 & 0.2(5) \\ \mathrm{C} 21-\mathrm{C} 20-\mathrm{C} 25-\mathrm{C} 24 & -1.0(5) \\ \mathrm{C} 7-\mathrm{C} 20-\mathrm{C} 25-\mathrm{C} 24 & 173.4(3) \\ & \end{array}$

$\begin{array}{ll}\mathrm{C} 10^{\prime}-\mathrm{C} 11^{\prime}-\mathrm{C} 12^{\prime}-\mathrm{C} 13^{\prime} & -1.1(6) \\ \mathrm{C} 11^{\prime}-\mathrm{C} 12^{\prime}-\mathrm{C} 13^{\prime}-\mathrm{C} 8^{\prime} & -0.1(6) \\ \mathrm{C} 9^{\prime}-\mathrm{C} 8^{\prime}-\mathrm{C} 13^{\prime}-\mathrm{C} 12^{\prime} & 1.2(5) \\ \mathrm{C} 7^{\prime}-\mathrm{C} 8^{\prime}-\mathrm{C} 13^{\prime}-\mathrm{C} 12^{\prime} & -178.0(3) \\ \mathrm{N} 2^{\prime}-\mathrm{C} 7^{\prime}-\mathrm{C} 14^{\prime}-\mathrm{C} 15^{\prime} & 130.6(3) \\ \mathrm{C} 8^{\prime}-\mathrm{C} 7^{\prime}-\mathrm{C} 14^{\prime}-\mathrm{C} 15^{\prime} & 16.5(4) \\ \mathrm{C} 20^{\prime}-\mathrm{C} 7^{\prime}-\mathrm{C} 14^{\prime}-\mathrm{C} 15^{\prime} & -103.9(3) \\ \mathrm{N} 2^{\prime}-\mathrm{C} 7^{\prime}-\mathrm{C} 14^{\prime}-\mathrm{C} 19^{\prime} & -52.4(3) \\ \mathrm{C} 8^{\prime}-\mathrm{C} 7^{\prime}-\mathrm{C} 14^{\prime}-\mathrm{C} 19^{\prime} & -166.5(3) \\ \mathrm{C} 20^{\prime}-\mathrm{C} 7^{\prime}-\mathrm{C} 14^{\prime}-\mathrm{C} 19^{\prime} & 73.0(3) \\ \mathrm{C} 19^{\prime}-\mathrm{C} 14^{\prime}-\mathrm{C} 15^{\prime}-\mathrm{C} 16^{\prime} & -2.8(5) \\ \mathrm{C} 7^{\prime}-\mathrm{C} 14^{\prime}-\mathrm{C} 15^{\prime}-\mathrm{C} 16^{\prime} & 174.2(3) \\ \mathrm{C} 14^{\prime}-\mathrm{C} 15^{\prime}-\mathrm{C} 16^{\prime}-\mathrm{C} 17^{\prime} & 1.6(6) \\ \mathrm{C} 15^{\prime}-\mathrm{C} 16^{\prime}-\mathrm{C} 17^{\prime}-\mathrm{C} 18^{\prime} & 0.5(6) \\ \mathrm{C} 16^{\prime}-\mathrm{C} 17^{\prime}-\mathrm{C} 18^{\prime}-\mathrm{C} 19^{\prime} & -1.2(5) \\ \mathrm{C} 17^{\prime}-\mathrm{C} 18^{\prime}-\mathrm{C} 19^{\prime}-\mathrm{C} 14^{\prime} & -0.1(5) \\ \mathrm{C} 15^{\prime}-\mathrm{C} 14^{\prime}-\mathrm{C} 19^{\prime}-\mathrm{C} 18^{\prime} & 2.1(5) \\ \mathrm{C} 7^{\prime}-\mathrm{C} 14^{\prime}-\mathrm{C} 19^{\prime}-\mathrm{C} 18^{\prime} & -175.0(3) \\ \mathrm{N} 2^{\prime}-\mathrm{C} 7^{\prime}-\mathrm{C} 20^{\prime}-\mathrm{C} 21^{\prime} & 130.7(3) \\ \mathrm{C} 8^{\prime}-\mathrm{C} 7^{\prime}-\mathrm{C} 20^{\prime}-\mathrm{C} 21^{\prime} & -109.3(3) \\ \mathrm{C} 14^{\prime}-\mathrm{C} 7^{\prime}-\mathrm{C} 20^{\prime}-\mathrm{C} 21^{\prime} & 12.6(4) \\ \mathrm{N} 2^{\prime}-\mathrm{C} 7^{\prime}-\mathrm{C} 20^{\prime}-\mathrm{C} 25^{\prime} & -55.4(4) \\ \mathrm{C} 8^{\prime}-\mathrm{C} 7^{\prime}-\mathrm{C} 20^{\prime}-\mathrm{C} 25^{\prime} & 64.6(3) \\ \mathrm{C} 14^{\prime}-\mathrm{C} 7^{\prime}-\mathrm{C} 20^{\prime}-\mathrm{C} 25^{\prime} & -173.5(3) \\ \mathrm{C} 25^{\prime}-\mathrm{C} 20^{\prime}-\mathrm{C} 21^{\prime}-\mathrm{C} 22^{\prime} & -1.3(5) \\ \mathrm{C} 7^{\prime}-\mathrm{C} 20^{\prime}-\mathrm{C} 21^{\prime}-\mathrm{C} 22^{\prime} & 172.7(3) \\ \mathrm{C} 20^{\prime}-\mathrm{C} 21^{\prime}-\mathrm{C} 22^{\prime}-\mathrm{C} 23^{\prime} & 0.3(5) \\ \mathrm{C} 21^{\prime}-\mathrm{C} 22^{\prime}-\mathrm{C} 23^{\prime}-\mathrm{C} 24^{\prime} & 1.4(5) \\ \mathrm{C} 22^{\prime}-\mathrm{C} 23^{\prime}-\mathrm{C} 24^{\prime}-\mathrm{C} 25^{\prime} & -2.0(5) \\ \mathrm{C} 23^{\prime}-\mathrm{C} 24^{\prime}-\mathrm{C} 25^{\prime}-\mathrm{C} 20^{\prime} & 0.9(5) \\ \mathrm{C} 21^{\prime}-\mathrm{C} 20^{\prime}-\mathrm{C} 25^{\prime}-\mathrm{C} 24^{\prime} & 0.7(5) \\ \mathrm{C} 7^{\prime}-\mathrm{C} 20^{\prime}-\mathrm{C} 25^{\prime}-\mathrm{C} 24^{\prime} & -173.5(3) \\ & \end{array}$

